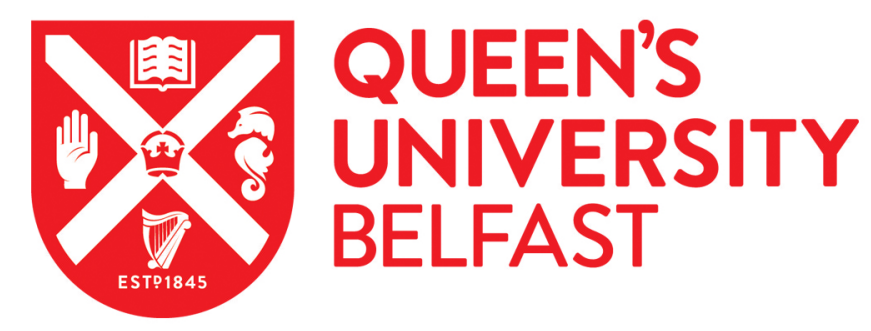

\title{
Hydrogels based on poly(methyl vinyl ether-co-maleic acid) and Tween 85 for sustained delivery of hydrophobic drugs
}

Larrañeta, E., Barturen, L., Ervine, M., \& Donnelly, R. F. (2018). Hydrogels based on poly(methyl vinyl ether-comaleic acid) and Tween 85 for sustained delivery of hydrophobic drugs. International Journal of Pharmaceutics, 1-42. https://doi.org/10.1016/j.ijpharm.2018.01.025

Published in:

International Journal of Pharmaceutics

Document Version:

Peer reviewed version

Queen's University Belfast - Research Portal:

Link to publication record in Queen's University Belfast Research Portal

Publisher rights

Copyright 2018 Elsevier.

This manuscript is distributed under a Creative Commons Attribution-NonCommercial-NoDerivs License

(https://creativecommons.org/licenses/by-nc-nd/4.0/), which permits distribution and reproduction for non-commercial purposes, provided the author and source are cited.

\section{General rights}

Copyright for the publications made accessible via the Queen's University Belfast Research Portal is retained by the author(s) and / or other copyright owners and it is a condition of accessing these publications that users recognise and abide by the legal requirements associated with these rights.

Take down policy

The Research Portal is Queen's institutional repository that provides access to Queen's research output. Every effort has been made to ensure that content in the Research Portal does not infringe any person's rights, or applicable UK laws. If you discover content in the Research Portal that you believe breaches copyright or violates any law, please contact openaccess@qub.ac.uk. 


\section{Accepted Manuscript}

Hydrogels based on poly(methyl vinyl ether-co-maleic acid) and Tween 85 for sustained delivery of hydrophobic drugs

Eneko Larrañeta, Laura Barturen, Michael Ervine, Ryan F. Donnelly

PII:

S0378-5173(18)30037-1

DOI: https://doi.org/10.1016/j.ijpharm.2018.01.025

Reference: IJP 17268

To appear in: Indian Journal of Pharmacology

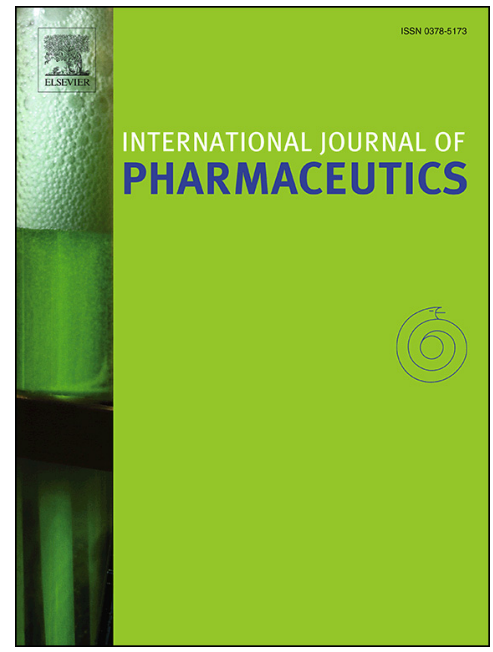

Received Date: 13 October 2017

Revised Date: $\quad 8$ January 2018

Accepted Date: $\quad 13$ January 2018

Please cite this article as: E. Larrañeta, L. Barturen, M. Ervine, R.F. Donnelly, Hydrogels based on poly(methyl vinyl ether-co-maleic acid) and Tween 85 for sustained delivery of hydrophobic drugs, Indian Journal of Pharmacology (2018), doi: https://doi.org/10.1016/j.ijpharm.2018.01.025

This is a PDF file of an unedited manuscript that has been accepted for publication. As a service to our customers we are providing this early version of the manuscript. The manuscript will undergo copyediting, typesetting, and review of the resulting proof before it is published in its final form. Please note that during the production process errors may be discovered which could affect the content, and all legal disclaimers that apply to the journal pertain. 
Hydrogels based on poly(methyl vinyl ether-co-maleic acid) and Tween 85 for sustained delivery of hydrophobic drugs.

Eneko Larrañeta*, Laura Barturen, Michael Ervine, Ryan F. Donnelly

School of Pharmacy, Queens University Belfast, Medical Biology Centre, 97 Lisburn Road, Belfast BT9 7BL, Northern Ireland, UK.

* Corresponding author

Dr. Eneko Larrañeta

Lecturer in Pharmaceutical Sciences

School of Pharmacy,

Queens University Belfast,

Medical Biology Centre,

97 Lisburn Road,

Belfast

BT9 7BL, UK

Tel: +44 (0) 2890972360

Fax: +44 (0) 2890247794

Email: e.larraneta@qub.ac.uk 


\section{Abstract}

Hydrogels based on poly(methyl vinyl ether-co-maleic acid) and Tween 85 were prepared for hydrophobic drug delivery. The hydrogels were synthesized following a simple procedure carried out in solid state. The process did not require the use of any solvent and, as it is based on an esterification reaction, no toxic by-products were obtained. The resulting hydrogels contained Tween 85 inside the structure and due to the amphiphilic nature of this compound, hydrophobic domains within the hydrogel structure were formed. The obtained hydrogels showed good swelling capacities ranging from $100 \%$ to $600 \%$. The esterification reaction that took place between poly(methyl vinyl ether-co-maleic acid) and Tween 85 was confirmed by infrared spectroscopy. Hydrogels were loaded with a hydrophobic drug model, Curcumin (CUR), showing that the hydrogels were able to retain up to $36 \mathrm{mg}$ of CUR per $\mathrm{g}$ of hydrogel. Additionally, the synthesized hydrogels provided in vitro sustained CUR release over periods of up to 30 days. Finally, and due to the mucoadhesive nature of the prepared materials, one of the hydrogels was tested in vitro as an oral drug delivery system. For this purpose, the selected material was milled into microparticles (45-90 $\mu \mathrm{m}$ diameter). The release of CUR from the microparticles was evaluated under simulated gastric and intestinal conditions. The microparticles were able to release their cargos in 7 hours. However, further work is required to optimize this system for oral drug delivery applications.

Keywords: Hydrogels; Hydrophobic drugs; Curcumin; Sustained Release 


\section{Introduction}

Hydrogels can be defined as three-dimensional networks of cross-linked polymers with a high capacity to absorb and retain water (Caló and Khutoryanskiy 2015; Hoare and Kohane 2008; Kopecek 2009; Larrañeta, et al. 2018; Peppas, et al. 2000). The crosslinking between the polymer chains can be based on chemical bonds (chemical hydrogels) or non-covalent interactions (physical hydrogels) (Caló and Khutoryanskiy 2015; Hoare and Kohane 2008). This type of materials can be prepared in a wide range of forms, such as micro/nano-particles, films, coatings or slabs among others (Hoare and Kohane 2008). Moreover, they can be produced using a wide range of polymers (Caló and Khutoryanskiy 2015; Hoare and Kohane 2008; Peppas, et al. 2000). As a result of this, hydrogels have been used extensively in clinical practice and experimental medicine for drug delivery, diagnostics or regenerative medicine (Caló and Khutoryanskiy 2015; Hoare and Kohane 2008; Peppas, et al. 2006).

Physical hydrogels have been extensively used to deliver a wide variety of compounds such as macromolecules (Branco and Schneider 2008; Cappello, et al. 1998; Larrañeta E, et al. 2014; Larrañeta and Isasi 2014), while chemical hydrogels as drug delivery systems are limited to the delivery of small and hydrophilic drugs (Hoare and Kohane 2008). One of the main limitations of this type of materials is their ability to load and release hydrophobic drugs (Hoare and Kohane 2008; McKenzie, et al. 2015). Due to their hydrophilic nature they present poor homogeneity and drug loading when combined with hydrophobic compounds (Hoare and Kohane 2008; McKenzie, et al. 2015).

Hydrophobic drugs are playing an important role in current pharmaceutical treatment and it is estimated that about $40 \%$ of the marketed drugs and $60 \%$ of the compounds at research and development state present poor water solubility (Fahr and Liu 2007; Gu, et al. 2016). Consequently, hydrogels should be adapted to be able to deliver this type of compounds successfully. Several strategies have been developed to try to overcome this limitation. Two of the main approaches are the introduction of molecules capable of forming inclusion complexes (ie. cyclodextrins) inside the hydrogel networks and the incorporation of hydrophobic moieties in the hydrogel structure (Concheiro and Alvarez-Lorenzo 2013; Gu, et al. 2016; McKenzie, et al. 2015).

Chemically-crosslinked hydrogels containing cyclodextrin moieties have been described extensively in the scientific literature (Concheiro and Alvarez-Lorenzo 2013; Crini and Morcellet 2002; Crini 2003; García-Zubiri, et al. 2009; Machín, et al. 2013; Machín, et al. 2011). The incorporation of cyclodextrin molecules covalently attached to the hydrogel backbone increased the capacity of the material to retain hydrophobic drugs. These systems showed the ability not only to be loaded with hydrophobic drugs but to release them over short periods of time (Machín, et al. 2011). 
A different strategy is to incorporate hydrophobic moieties to the hydrophilic polymers that forms the hydrogel (McKenzie, et al. 2015). This type of hydrogels are commonly physical-hydrogels (Larrañeta and Isasi 2012; McKenzie, et al. 2015). A simple way of obtaining physical gels is to attach a hydrophobic moiety to a hydrophilic polymer. Due to hydrophobic interactions, the system will aggregate forming a physical gel. This type of system includes hydrophobic regions inside the hydrogels. This regions can be used to load hydrophobic drugs effectively (McKenzie, et al. 2015).

In the present work, the synthesis, characterization and evaluation of chemically crosslinked hydrogels containing hydrophobic moieties is presented. For this purpose, poly(methyl vinyl etheralt-maleic acid) was covalently crosslinked by using an ampiphilic molecule, Tween $85^{\circledR}$ (T85). The synthesis was developed in solid state using a thermal process that can be easily replicated and scaled up. Further, the developed hydrogels present potential as a system for hydrophobic drug delivery over prolonged periods of time. Curcumin (CUR) was used as a hydrophobic drug molecule model in order to evaluate the loading and release capacities of the synthesized hydrogels. The obtained results showed that T85 containing hydrogels have the potential to be used for prolonged delivery of hydrophobic drugs in applications such as drug eluting implants.

\section{Material and methods}

\subsection{Materials}

Gantrez $^{\circledR}$ S-97 (GAN) (acid form of methylvinylether and maleic anhydride copolymer) $(\mathrm{Mw}=1.2 \times 106 \mathrm{Da})$, was provided by Ashland (Tadworth, UK). T85 was obtained from Croda (Snaith, United Kingdom). CUR was purchased from Cambridge Bioscience (Cambridge, United Kingdom).

\subsection{Particle Size analysis of T85 solutions}

T85 water solutions were analysed using a Nano ZS Zetasizer and DTS software (Malvern Instruments, UK) at $25^{\circ} \mathrm{C}$. Each measurement $(\mathrm{n}=3)$ had 10 sub measurements.

\subsection{Preparation of the hydrogels}

Aqueous solutions containing different ratios of T85 and GAN were prepared and 30g of these solutions were casted in $10 \times 10$ moulds. Solutions were allowed to dry over at least 48 hours. The resulting films were cut in pieces of $1 \times 1 \mathrm{~cm}$ and subsequently placed inside an oven at $80^{\circ} \mathrm{C}$ for 24 
hours. The hydrogels were named after the composition of the aqueous blends used to prepare the films (Table 1). GAN-based hydrogels were prepared by crosslinking the polymer with poly(ethylene glycol) (PEG) $\left(M_{w}=10,000\right)$ following the same protocol. These hydrogels were prepared using the previously optimized formulation described by Raj Singh et al (Raj Singh, et al. 2009).

\subsection{Hydrogel characterization}

Films $(1.0 \times 1.0 \mathrm{~cm})$ were weighed as $\mathrm{m}_{\mathrm{o}}$ and then swollen in water for 24 hours at room temperature. At regular intervals, the films were removed, dried with filter paper to eliminate excess surface water and weighed as $m_{t}$ (hydrogels). The percentage swelling, was calculated, respectively, by using Equation 1.

$\%$ Swelling $=\frac{m_{t}-m_{o}}{m_{o}} \times 100$

The same procedure was followed to study the swelling of hydrogels in $\mathrm{pH} 7$ Phosphate Buffer Solution (PBS) and in $\mathrm{HCl}$ aqueous solution $0.12 \mathrm{M}$.

The average molecular weight between crosslinks $\left(M_{c}\right)$ was determined using equilibrium swelling theory. $M_{c}$ can be determined by swelling studies according to the Flory and Rehner equation (Equation 3) (2) (Flory and Rehner 1943). All these calculations were carried out with the results obtained after swelling the hydrogels in water over a 24 hour timeframe.

$$
M_{c}=\frac{-\mathrm{d}_{\mathrm{p}} V_{S} \phi}{\left(\ln (1-\phi)+\phi+\chi \phi^{2}\right)}
$$

The volume fraction of a polymer, $\phi$, in the swollen state describes the amount of liquid that can be imbibed into a hydrogel and is described as a ratio of the polymer volume to the swollen gel volume (Equation 3). Vs is the molar volume of water $\left(18 \mathrm{~cm}^{3} / \mathrm{mol}\right), \chi$ is the Flory-Huggins polymer-solvent interaction parameter. This parameter $(\chi)$ reflects the thermodynamic interaction in hydrogels, which in turn indicates the change of interaction energy when polymer and solvent mix together. The $\chi$ parameters of hydrogels can be calculated using Equation 4 (Çaykara, et al. 2006).

$$
\begin{aligned}
& \phi=\left[1+\frac{d_{p}}{d_{s}}\left(\frac{m_{a}}{m_{b}}\right)-\frac{d_{p}}{d_{s}}\right]^{-1} \\
& \chi=\frac{1}{2}+\frac{\phi}{3}
\end{aligned}
$$


In order to calculate $\phi$ using Equation 3, the following parameters were used: mass of hydrogel before $\left(m_{b}\right)$, the mass of the hydrogel after swelling $\left(m_{a}\right)$, the density of the hydrogel $\left(d_{p}\right)$ and the density of the solvent $\left(d_{s}\right)$. The density of the hydrogel films was calculated using Equation 5.

$$
d_{p}=\frac{w}{S \times X}
$$

Where $X$ is the average thickness of the film, $S$ is the cross-sectional area and $w$ weight of the film (Raj Singh, et al. 2009).

The crosslink density $\left(V_{e}\right)$ was determined using Equation 6. $V_{e}$ represents the number of elastically effective chains, totally induced in a perfect network, per unit volume (Bajpai and Singh 2006; Raj Singh, et al. 2009). Where $N_{A}$ is Avagadro's number $\left(6.023 \times 10^{23} \mathrm{~mole}^{-1}\right)$

$$
V_{e}=\frac{d_{p} \times N_{A}}{M_{c}}
$$

Attenuated total reflectance (ATR)-Fourier transform infrared (FTIR) spectroscopy was used to evaluate the esterification reaction between GAN and T85. The IR spectra were recorded at room temperature using a FTIR Accutrac FT/IR-4100 Series (Jasco, Essex, UK) equipped with MIRacle ${ }^{\mathrm{TM}}$ software between $4000-600 \mathrm{~cm}^{-1}$ with a resolution of $4.0 \mathrm{~cm}^{-1}$. The obtained spectra were the result of averaging 64 scans.

Samples were analyzed at room temperature using a MiniFlex II desktop powder X-ray diffractometer (Rigaku Corporation, Kent, England) equipped with $\mathrm{Cu} K \beta$ radiation, at a voltage of 30 $\mathrm{kV}$ and a current of $15 \mathrm{~mA}$. All samples were scanned within the angular range of $1.5-50^{\circ} 2 \theta$ in continuous mode.

The morphology of the hydrogels was evaluated by using electronic and optical microscopy. A Hitachi TM3030 benchtop scanning electron microscope (SEM) (Tokyo, Japan) was used. Samples were analysed after swelling in water and after being freeze dried. Samples were measured directly without any sample preparation. The morphology of the hydrogels was evaluated using a Leica EZ4 D digital microscope (Leica, Wetzlar, Germany). Hydrogels were observed in the swollen state and in the dry state after freeze drying the swollen hydrogels

\subsection{Curcumin loading and release}

All the hydrogels were loaded with CUR by immersing the dry film in $1 \mathrm{~mL}$ of a $20 \mathrm{mg} / \mathrm{mL}$ solution of CUR in acetone. The film was left inside the solution for 24 hours. After the loading process films 
were removed, dried with filter paper to eliminate the superficial excess of CUR solution. In order to evaluate the loading capacity of the hydrogels, the loaded films were placed in $20 \mathrm{~mL}$ of ethanol over a 24 hour timeframe until all the CUR was released. The concentration of CUR in the ethanol was evaluated using a UV-visible plate reader (PowerWave XS Microplate Spectrophotometer, BioTek, Winooski, USA) at a wavelength of $425 \mathrm{~nm}$. The experiment was done in triplicate.

CUR loaded films were placed cut in 4 fragments and a one of the fragments was placed in a tube containing $10 \mathrm{~mL}$ of PBS (pH 7.3) with $10 \%$ of Tween 80 to maintain sink conditions. The release media contains $1 \mathrm{mg} / \mathrm{mL}$ of ascorbic acid to prevent CUR oxidation. All the tubes were placed in a shaking incubator $\left(40 \mathrm{rpm}\right.$ and $\left.37^{\circ} \mathrm{C}\right)$. For comparison purposes, the dissolution of pure CUR powder in the release media was evaluated. CUR powder (ca. $1.2 \mathrm{mg}$ ) were placed in a tube containing $12 \mathrm{~mL}$ of PBS (pH 7.3) with $10 \%$ of Tween 80 and $1 \mathrm{mg} / \mathrm{mL}$ of ascorbic acid.

Ascorbic acid was required to obtain consistent results for the CUR quantification method development. Without this compound the calibration curves presented high variability. This can be observed in Figures S1A and S1B (supporting information). Additionally, the stability of CUR in the release medium with and without ascorbic acid was studied. The results (see supporting information Figure S1C) showed that CUR was stable in both media for at least around $24 \mathrm{~h}$. However, the CUR concentration obtained in the medium containing ascorbic acid were less variable. The combination of Tween 80 and ascorbic acid provided high stability to CUR in solution. This crucial as it has been reported previously that CUR is highly unstable in aqueous environment (Kharat, et al. 2017).

At certain times the release medium was replaced with fresh one and the concentration of CUR was evaluated using a UV-visible plate reader at a wavelength of $425 \mathrm{~nm}$. For CUR powder control, at defined times a samples (0.02-0.05 $\mathrm{mL}$ ) were collected to evaluate the concentration of CUR dissolved and they were replaced by fresh medium.

Films loaded with CUR were analysed using infrared spectroscopy (see above) and a TA Instruments DSC Q100 differential scanning calorimeter (DSC Q100). Samples were analysed from 0 to $200^{\circ} \mathrm{C}$ at a heating speed of $10^{\circ} \mathrm{C} / \mathrm{min}$.

\subsection{Analysis of release data.}

Different parameters were calculated with the CUR release data obtained from the hydrogels. The mean dissolution time (MDT) can be calculated using Equation 7 (Costa, et al. 2001). 
$M D T=\frac{\sum_{j=1}^{n} \hat{t}_{j} \cdot \Delta M_{j}}{\sum_{j=1}^{n} M_{j}}$

where $\mathrm{j}$ is the sample number, $\mathrm{n}$ is the number of dissolution sample times, $\hat{t}_{j}$ is the time at the midpoint between $t_{j}$ and $t_{j-1}$ and $\Delta M_{j}$ is the additional amount of drug dissolved between $t_{j}$ and $t_{j-1}$.

The dissolution efficiency (DE) of a pharmaceutical dosage form is defined by the percentage of area under the dissolution curve up to a certain time $t$ expressed as a percentage of the area of the rectangle described by $100 \%$ dissolution $\left(\mathrm{y}_{100}\right)$ in the same time (Equation 8 ) (Costa, et al. 2001). It is important to notice that in this case the formulation is not dissolved as it is a hydrogel, however we have used this parameter in order to compare CUR release from all the different types of hydrogels.

$D E=\frac{\int_{0}^{t} y \times d t}{y_{100} \times t} \times 100$

In order to compare different release curves, the difference $\left(f_{1}\right)$ and similarity factor $\left(f_{2}\right)$ were calculated (Costa, et al. 2001). The difference factor $\left(f_{1}\right)$ (Equation 9) calculates the percent (\%) difference between the two curves at each time point and is a measurement of the relative error between the two curves: where $\mathrm{n}$ is the number of time points, $\mathrm{R}$ is the dissolution value of the reference $t$ (prechange) batch at time $t$, and $T$ is the dissolution value of the test (postchange) batch at time $t$.

$$
f_{1}=\left\{\left[\sum_{j=1}^{n}\left|R_{t}-T_{t}\right|\right] /\left[\sum_{j=1}^{n} R_{t}\right]\right\} \cdot 100
$$

The similarity factor $\left(f_{2}\right)$ (Equation 10) is a logarithmic transformation of the sum-squared error of differences between the test $T_{j}$ and reference products $R_{j}$ over all time points, $n$ (Costa, et al. 2001).

$$
f_{2}=50 \cdot \log \left\{\left[(1 / n) \sum_{j=1}^{n}\left(R_{t}-T_{t}\right)^{2}\right]^{-0,5} \cdot 100\right\}
$$

The data obtained from the in vitro release experiments were fitted to different mathematical models of drug release. The models used for this study were the Korsmeyer-Peppas model (Equation 11) and the Higuchi model (Equation 12).

The Korsmeyer-Peppas model exponentially relates drug release with the elapsed time (Costa, et al. 2001; Ritger and Peppas 1987). 
$\frac{M_{t}}{M_{\infty}}=\mathrm{k}_{K P} \cdot \mathrm{t}^{\mathrm{n}}$

where $M_{t} / M_{\infty}$ is the drug release fraction at time $t, k_{K P}$ is a constant incorporating the structural and geometric characteristics of the matrix tablets, and $\mathrm{n}$ is the release exponent indicative of the drug release mechanism. The value of $\mathrm{n}$ indicates the mechanism of the release. 27 If the value is around 0.5 (the exact value depends of the geometry) the mechanism is Case I (Fickian) diffusion and a value between 0.5 and 0.89 indicates anomalous (non-Fickian) diffusion. Values of $\mathrm{n}$ equal to 0.89 indicate Case II transport.

If the obtained release mechanism is mainly a Fickian diffusion, the Higuchi model was used (Costa, et al. 2001) (Equation 12). In this case $M_{t} / M_{\infty}$ is the drug release fraction at time $t$ and $k_{H}$ is the Higuchi constant. In the original Higuchi equation, the $M_{t}$ (amount of drug released at time $t$ ) term is found instead of $M_{t} / M_{\infty}$.

$\frac{M_{t}}{M_{\infty}}=\mathrm{k}_{H} \cdot \mathrm{t}^{0.5}$

The zero-order kinetics equation was also used in order to evaluate the type of release mechanism. This model is used for systems where the matrix releases the same amount of drug by unit of time (Costa, et al. 2001). This model relates the amount of drug dissolved with time. However, we have used a modified zero-order equation, where we have replaced the amount or drug released term by $M_{t} / M_{\infty}$ (Equation 13).

$\frac{M_{t}}{M_{\infty}}=\mathrm{k}_{\mathrm{zO}} \cdot \mathrm{t}$

In this case $M_{t} / M_{\infty}$ is the drug release fraction at time $t$ and $k_{z o}$ is the Zero-Order constant.

\subsection{In vitro mucoadhesion study}

The mucoadhesive properties of the hydrogels were evaluated using a TA-XT2 Texture Analyser (Stable Micro Systems, Surrey, UK). Mucin discs were manufactured by compression of mucin (500 $\mathrm{mg}$ ) using a ring press with a $13 \mathrm{~mm}$ die and a compression force of 10 tonnes, applied for $30 \mathrm{~s}$. These were attached to an aluminium block using double sided adhesive tape. Hydrogel films were attached to the equipment probe with double side adhesive tape. Before starting the test, around $200 \mu \mathrm{L}$ of a mucin solution was applied to the surface of the mucin disc and after $30 \mathrm{~s}$ it was blotted with tissue paper. A defined force of $0.1 \mathrm{~N}$ was applied for $30 \mathrm{~s}$ to ensure intimate contact between 
the mucin disc and the sample. The probe was then moved vertically upwards at a constant speed of $1.0 \mathrm{~mm} \mathrm{~s}^{-1}$ and the force required to detach the mucin disc from the surface of each formulation was determined from the resultant force-time plot. All measurements were performed, at least, in quadruplicate.

\subsection{Release of CUR from hydrogel microparticles}

Hydrogel microparticles (MPs) were prepared by milling 5 T85 hydrogels in a coffee grinder. The obtained powder was sieved and the fraction with sizes ranging between 45 and $90 \mu \mathrm{m}$ were collected. The morpohology of the particles was observed using SEM (Hitachi TM3030).

The particles were loaded following the same protocol described for the loading of the hydrogels. Between $100-200 \mathrm{mg}$ of the particles were placed in $2 \mathrm{~mL}$ of a CUR acetone solution $(20 \mathrm{mg} / \mathrm{mL})$. After 24 hours the particles were isolated by filtration and washed with acetone in order to remove the excess of CUR loaded. The CUR loading was evaluated by placing $2.5 \mathrm{mg}$ of the particles in $2 \mathrm{~mL}$ of ethanol. After 24 hours, all the CUR contained in the particles was released and the concentration was evaluated as described before.

The release kinetics of CUR loaded microparticles was evaluated simulating the gastrointestinal tract conditions. During the first two hours the release medium was $\mathrm{HCl} 0.12 \mathrm{M}$ simulating gastric conditions. Subsequently the release medium was changed to PBS to simulate intestinal conditions. In all cases the release media contained ascorbic acid $(1 \mathrm{mg} / \mathrm{mL})$ to prevent CUR degradation. At certain times the release media was replaced with fresh one and the amount of released CUR was evaluated by using UV-visible spectroscopy.

For comparison purposes, the dissolution of pure CUR powder in the release media was evaluated. CUR powder (ca. $1 \mathrm{mg}$ ) were placed in a tube containing $20 \mathrm{~mL}$ of simulated gastric solution and simulated intestinal solution. At defined times a samples $(0.02-0.05 \mathrm{~mL})$ were collected to evaluate the concentration of CUR dissolved and they were replaced by fresh medium.

\subsection{Statistical Analysis}

Where appropriate, data was analyzed using a one-way ANOVA with post hoc comparisons performed using the Tukey-Kramer test. In all cases, $p<0.05$ denoted significance. Statistical Package for the Social Sciences, SPSS 18.0 version 2.0 (SPSS, Inc., Chicago, IL, USA), was used for all analyses. 


\section{Results}

\subsection{Hydrogel characterization}

GAN has been used in the past to prepare hydrogels through esterification reactions (Larrañeta, et al. 2015; Moreno, et al. 2014). T85 is an ideal candidate to introduce hydrophobic domains in the hydrogel structure. This surfactant present a complex structure containing oleic acid chains and alcohol groups (Solak, et al. 2014). The alcohol groups can be used to crosslink GAN chains by reaction with the acid groups present in GAN molecules.

Figure $1 \mathrm{~A}$ shows the size distribution of $\mathrm{T} 85$ solutions in water. It can be seen that surfactant forms aggregates with different sizes ranging from $10 \mathrm{~nm}$ to $1 \mu \mathrm{m}$. The size distribution changes as a function of the concentration. These results showed the tendency of T85 to form aggregates in aqueous environments due to its amphiphilic nature. All the solutions presented a certain degree of turbidity confirming this tendency. Consequently, we decided that T85 was a good candidate to be incorporated within hydrogels to add hydrophobic domains to the structure.

Figure 1B shows the swelling profiles in water of all synthesized hydrogels. As can be seen in Figure $1 B$, hydrogels containing higher concentrations of T85 showed lower swelling capacities $(p<0.05)$. Higher concentrations of T85 in the materials yielded hydrogels with higher crosslinking degrees and consequently, lower swelling capacity. In order to compare GAN/T85 hydrogels with a similar hydrogel that does not contain hydrophobic domains, P2 hydrogel was used as control. This hydrogel has been used extensively in the past for drug delivery purposes (Donnelly, et al. 2012; Larrañeta, et al. 2015; Raj Singh, et al. 2009). This control hydrogel showed a similar swelling profile compared to T3.

Table 2 shows the network parameters obtained for all the different hydrogels. The volume fraction of polymer in the swollen state $(\phi)$ represents the amount of liquid that can be absorbed by an hydrogel (Raj Singh, et al. 2009). As can be seen in Table 2, this parameter decreased when the concentration of T85 in the hydrogels increased. Furthermore, the average molecular weights between crosslinks, $M_{c}$ (Equi), increases with the concentration of T85 in the hydrogel.

The polymer-water interaction parameter $(\chi)$ is proportional to the crosslink density. The higher the value of $\chi$, the weaker is the interaction between the polymer and water. Therefore, increasing the crosslinking degree increased the interaction between the polymeric system and water. 
Furthermore, $\mathrm{V}_{\mathrm{e}}$ is another good indicator of the crosslinking density. It represents the number of elastically effective chains, totally induced in a perfect network, per unit volume (Bajpai and Singh 2006; Raj Singh, et al. 2009). Consequently, hydrogels with higher crosslinking densities presented higher $V_{e}$. The calculated $V_{e}$ values are consistent with the results obtained in the swelling studies: hydrogels with higher amount of T85 showed higher crosslinking degrees. Finally, it can be seen that all the calculated network parameters for P2 and T3 are similar. This is consistent with the results described previously.

The presence of T85 within the hydrogel structure influenced the swelling of the material when using alternative solvents. The study of the absorption of organic solvents by the hydrogels is a good way to ascertain the hydrophobicity of the materials. Figure $1 \mathrm{C}$ shows the maximum swelling of the hydrogels in water, acetone and ethanol. Additionally, Figure 1D shows the maximum swelling of the hydrogels as a function of the relative polarity of the solvent. P2 hydrogels showed lower swelling capacity in acetone than the GAN/T85 hydrogels $(p<0.05)$ (Figure 1C). However, it showed similar ethanol uptake to T5 $(p>0.05)$. The solvent uptake in this case is proportional to the relative polarity of the solvent (Figure 1D) showing that the highest swelling was obtained in water and the lowest in acetone. On the other hand, T85 containing hydrogels presented a different behaviour (Figure 1C). T2 hydrogels showed a higher swelling capability in water than in ethanol $(p<0.05)$. On the other hand, hydrogels containing higher concentrations of T85 showed comparable swelling capacity in ethanol and water $(p>0.05)$. The higher ethanol uptake for hydrogels containing T85 can be explained by the presence of oleic acid chains in the structure of the surfactant. This fatty acid presents unlimited solubility in ethanol at ambient temperature (Hoerr and Harwood 1952) and consequently the interactions of T85 with ethanol are energetically favourable. Besides, the swelling in ethanol follows the same trend obtained in water: higher swelling degrees were obtained for hydrogels with lower T85 concentrations. This indicates that the affinity of T85 and ethanol is not the only limiting factor as the crosslinking degree of the hydrogel plays a more important role.

The swelling capacity of these materials in acetone was lower than the one obtained in ethanol $(p<$ $0.05)$ with the exception of T5 $(p=0.999)$. Interestingly, in this case the higher swelling in acetone was obtained for T5. Despite having higher crosslinking degrees, the presence of higher amounts of T85 in its structure allows a higher acetone uptake. This can be explained by the solubility of oleic acid in acetone. Hoerr et al. showed that the solubility of this fatty acid in acetone was unlimited at ambient temperature (Hoerr and Harwood 1952). Consequently, there are favourable interactions between the solvent and the hydrophobic moieties of T85. This trend can be seen in Figure 1D as it demonstrates that hydrogels containing higher quantities of T85 showed better absorption of 
solvents with lower polarity. The ability to uptake organic solvents is really useful for drug loading applications, as it is a good way to load hydrophobic drugs inside the hydrogel.

Figure 2 shows SEM images of the hydrogels in the swollen state and after being freeze dried. It can be seen that all the materials showed a compact structure when swollen. It is important to note that the images were captured using an environmental SEM and the samples should be placed in a vacuum chamber. Consequently, it is quite likely that the swollen hydrogels lose water during the process. The presence of pores in T5 hydrogels can be observed. These pores are generated by trapped air bubbles. Due to the viscosity of the aqueous mixtures used to prepare T5 films, small air bubbles were trapped in the structure.

After the freeze drying process, the structure of the hydrogels showed high porosity. The size of the pores can be related with the swelling capacity of the hydrogels. The pores were smaller in T5 while higher pore sizes were evident in T3 and T2. Interestingly, P2 hydrogels showed a smaller pore size distribution than T3 hydrogels. Both hydrogels showed similar water uptake capabilities but their internal structure after swelling showed some differences. This can be explained by the presence of the surfactant in T3 generating hydrophobic domains inside the hydrogel.

Hydrogels were analysed by using FTIR spectroscopy. Figure 3A shows the spectra of P2 and T3 before and after the crosslinking reaction. The main difference between the crosslinked and noncross-linked (NC) materials can be found in the carbonyl region. After the crosslinking process different reactions took place: esterification between T85/PEG and GAN and anhydride formation between two GAN acid groups (Larrañeta, et al. 2015). The esterification can be ascertained by the shift of the carbonyl peak at around $1700 \mathrm{~cm}^{-1}$. The infrared carbonyl peaks for carboxylic acids and esters are overlapping (Sclavons, et al. 2000). The observed peak shift suggests the presence of a new ester peak overlapping with the previous acid peak (Sclavons, et al. 2000). Additionally, a new peak appears at around $1780 \mathrm{~cm}^{-1}$, due to the formation of anhydride groups between two acid groups in the GAN molecule (Larrañeta, et al. 2015; Sclavons, et al. 2000). The anhydride formation can be ascertained by the presence of two new peaks between 1750 and $2000 \mathrm{~cm}^{-1}$. Figure 3B shows the FTIR spectra of all the crosslinked hydrogels. It is important to note that the peak attributed to ester carbonyl presented higher relative intensity in T5 hydrogels and the lower in T2 hydrogels. This is consistent with the swelling results suggesting that the crosslinking degree is proportional to the T85 concentration. The similar peak shape for P2 and T3 hydrogels reinforces the results obtained in the swelling studies.

The presence of amphiphilic molecules within the hydrogel structure will induce the formation of hydrophobic domains. $\mathrm{X}$ ray diffraction (XRD) was used to evaluate the internal structure of the 
hydrogels. Figure 4A shows the XRD pattern obtained for all the synthesized hydrogels. The diffraction patter of all the hydrogels, including P2, showed a broad peak suggesting an amorphous structure. However, the peak is sharper for the hydrogels prepared using higher concentrations of T85. This suggest an increase in the internal order. The area and the full width at half maximum (FWHM) values of the diffraction peaks are related to the crystallinity of the material (Parthiban, et al. 2006). Consequently, the FWHM of these peaks was measured (Figure 4B). The obtained values can be correlated with the T85/GAN ratios suggesting that hydrogels containing higher amounts of T85 presented lower FWHM, and consequently a higher degree of internal order. This results suggest that the presence of T85 in the structure contributes to the internal order or the hydrogels. Consequently, this internal order should be a result of the internal hydrophobic domains generated by the presence of $\mathrm{T} 85$.

Figure 5 shows images of the hydrogels before and after hydration. It can be seen that the hydrogels presented certain degree of turbidity when swollen. The materials showed a similar behaviour than the T85 solutions. The results suggest that T85 is forming hydrophobic domains inside the hydrogel structure. On the other hand, P2 hydrogels did not show any turbidity when swollen as this type of hydrogels does not present hydrophobic domains (data not shown).

\subsection{Curcumin release studies.}

CUR is an hydrophobic polyphenol with hypoglycemic, antioxidant, wound-healing, antiinflammatory and antimicrobial activities (Aggarwal and Sung 2009). Therefore, it is an ideal candidate as a model compound to study the loading and release capabilities of the synthesized hydrogels. Figure $6 \mathrm{~A}$ shows the chemical structure of CUR.

Table 3 shows the CUR loading capacity of the hydrogels. It is important to note that hydrogels containing higher amounts of T85 presented higher loading capacities. Hydrogels were loaded by placing them in a CUR acetone solution. The loading results can be correlated with the swelling of the hydrogels in acetone and with the \%T85 in the hydrogel (see supporting information Figure S2). Consequently, the addition of hydrophobic moieties to the hydrogel improves significantly hydrophobic drug loading. Moreover, it is important to note that there was no significant difference between T3 and T5 CUR loading $(p=0.958)$. This suggest that the system reached a maximum CUR uptake as increasing the concentration of T85 in these hydrogels did not translated into a higher drug loading. 
FTIR and DSC were used to analyse CUR containing hydrogels. Figure 6B shows the FTIR spectra of pure CUR and the hydrogels before and after being loaded with this molecule. The result showed that the peak attributed to the CUR carbonyl group $\left(1500 \mathrm{~cm}^{-1}\right)$ (Mohan, et al. 2012) can be seen in all the loaded hydrogels. A peak shift can be observed suggesting that CUR molecules interact with the hydrogel structure. The shift is more noticeable for T85 containing hydrogels. Similar behaviour has been reported by Mohan et al. for the complexation of CUR with cyclodextrins (Mohan, et al. 2012).

Figure 6C shows the DSC curves obtained for CUR and the loaded hydrogels. In all cases, the CUR melting peak (ca. $180^{\circ} \mathrm{C}$ ) cannot be seen in the DSC curves of the hydrogels. Similar results were obtained for CUR/cyclodextrin complexes (Mohan, et al. 2012). This suggest that CUR molecules are dispersed in the hydrogel matrix. Therefore, the results obtained from FTIR and DSC suggest that the drug is interacting with the polymeric chains of the hydrogel.

The release of CUR from the synthesized hydrogels can be seen in Figure 7A. It is important to note that the mass of the hydrogels used in these experiments was slightly different for every type of hydrogel as a piece of film was divided in 4 (see material and methods section). Consequently, and in order to compare the release profiles properly, Figure 7C was included showing the $\%$ of the loaded CUR released as a function of time. P2 hydrogels showed the fastest CUR release (Figure 7B). This type of hydrogels released all the CUR cargo within 1 day. On the other hand, T85 containing hydrogels showed a prolonged CUR release over a period of 30 days. T2 and T3 showed similar release profiles. On the other hand, T5 hydrogels presented a more sustained release profile. The results demonstrate that the presence of T85 in the hydrogels allows sustained releases of CUR. Figure 7D shows the dissolution profile of CUR in the release medium. For this experiment the selected amount of CUR was adapted to be similar to the amount present inside the hydrogels. As the experiment was performed under sink conditions after 3-4 hours all the CUR powder was dissolved. Consequently, we can consider that hydrogels are responsible for the sustained release profiles.

In order to compare the obtained release profiles, DE, MDT and $f_{1}$ and $f_{2}$ were calculated (Table 4). CUR released from P2 hydrogels showed the highest DE values. Hydrogels containing T85 showed a decrease in the DE proportional to the T85 content. This indicates that the presence of more hydrophobic domains inside the hydrogels slows down CUR release process. The MDT results showed that P2 provided the quickest release of 50\% of the CUR cargo. T2 and T3 showed similar MDT values. However, according to these results, T2 showed a slightly slower release. Finally, T5 showed the highest MDT values. 
In order to compare all the release curves $f_{1}$ and $f_{2}$ factors were calculated (Table 4). When comparing two release curves, if the calculated $f_{1}$ values are lower than 15 and the values of $f_{2}$ are higher than 50 both curves can be considered equivalent. According to this, T2 and T3 showed equivalent CUR release profiles. However, in the next paragraphs it will be discussed that despite having equivalent CUR release capacity, the release mechanism is slightly different.

Table 5 shows the results obtained after fitting the release data to the Korsmeyer-Peppas and Higuchi model. The Korsmeyer-Peppas model was used to calculate the main mechanism of drug release. The obtained " $n$ " values ranged between 0.45 and 0.55 , suggesting that the CUR release from the hydrogels is governed by Fickian diffusion (Larrañeta E, et al. 2014; Ritger and Peppas 1987).

Higuchi model was used as it is a model designed for drug delivery systems with release governed by pure Fickian diffusion. As can be seen in Table 5, the $\mathrm{R}^{2}$ values indicating good fitting to the Higuchi model. These results are consistent with the results obtained before, suggesting that the main release mechanism of CUR from the all the hydrogels is Fickian diffusion.

It is important to note that despite of presenting similar release profiles when calculating $f_{1}$ and $f_{2}$ factors, T2 shows a different release profile than T3. T3 showed a good fitting to the Higuchi model however $\mathrm{T} 2$ curve shows a biphasic release pattern as 5 days after starting the release process the curves showed a linear release (Zero Order kinetic). This process continued between the day 5 and the day 24. Consequently, the Korsmeyer-Peppas and Higuchi models were applied again to the T2 release data obtained within the first 3 days of release instead of the previous first 7 days $(\leq 60 \%$ release) (Table 5). The obtained results showed better fitting to both equations, suggesting that during the first 3 days the release process was governed by a diffusion mechanism. Moreover, a Zero Order model was applied to the next section of the release curve (5-24 days) showing good correlation with the model (Table 5). After a few days from the beginning of the release experiment some opaque domains were formed within $\mathrm{T} 2$ structure that disappeared over time. This observation suggests that CUR crystallization within the hydrogel structure. The crystallization of CUR in T2 can be explained by the lower amount of T85 in these hydrogels to stabilize CUR preventing crystal growth when compared with $\mathrm{T} 2$ and $\mathrm{T} 3$. These factor can explain the difference in the release mechanism for $\mathrm{T} 2$.

\subsection{Tween 85-based hydrogels as oral drug delivery systems.}


GAN polymers present a large number of acid groups that can form hydrogen bonds. Therefore, this type of polymers has been used extensively to prepare mucoadhesive systems for oral drug delivery (Agüeros, et al. 2011; Arbós, et al. 2002; Ojer, et al. 2013). Mucoadhesive drug delivery systems interact with the mucus layer of the gastrointestinal tract, increasing the residence time of the dosage form at the site of absorption (Boddupalli, et al. 2010). Additionally, due to the presence of the acid groups, GAN provides $\mathrm{pH}$ dependant drug delivery showing different release rates under gastric and intestinal conditions (Agüeros, et al. 2009).

Due to the presence of GAN in the hydrogels, the materials described in this work have the potential to be used as oral mucoadhesive drug delivery systems. Figure 8 shows the mucoadhesion test of the all the hydrogels studied in the present work. Despite the different adhesion force obtained for P2, T2 and T3 hydrogels, there is no significant differences in their mucoadhesivity ( $p>0.05$ ). However, T5 showed a lower adhesion force than T3 $(p=0.012)$. T5 has a higher crosslinking degree than T3, and consequently, it has lower free acid groups to take part in the adhesion process. The obtained results suggest that the adhesion forces are higher than the ones obtained by Calo et al. for a similar type of hydrogels (Calo, et al. 2016b).

All the hydrogels showed good mucoadhesivity but T2 and T3 were selected for further studies due to their drug delivery capabilities. T5 was discarded as the drug release from this type of hydrogels was considered too slow for oral drug delivery systems. The swelling behaviour of T2 and T3 at different pHs, simulating gastric and intestinal conditions, were tested (Figure $8 \mathrm{~B}$ ). It can be seen that, due to the presence of multiple acid groups in the hydrogel, both hydrogels showed lower swelling at lower acidic pHs. Higher $\mathrm{pH}$ causes dissociation of $\mathrm{COOH}$ groups of $\mathrm{GAN}$, preventing intermolecular $-\mathrm{COOH} \cdots \mathrm{HO}$ - bonds and, consequently, yielding higher swelling degrees (Calo, et al. 2016a). T2 hydrogels showed a larger swelling difference between the tested pHs. Consequently, this type of hydrogels was selected for further studies.

The results presented in Figure 7 suggested that the release from T85 based hydrogels is slow. Consequently, we decided to reduce the size of the hydrogels to accelerate the drug release process. MPs of T2 were obtained by milling hydrogel films. Moreover, a powder formulation is a better alternative for oral drug delivery than hydrogel films, as the MPs powder can be easily encapsulated or compressed to obtain tablets. The MPs presented sizes ranging between 45 and $90 \mu \mathrm{m}$. Figure $9 \mathrm{~A}$ shows SEM images of the obtained particles. The particles were loaded with CUR, obtaining a loading of $41 \pm 7 \mathrm{mg}$ CUR/g hydrogel.

Figure 9B shows the release of CUR from the MPs in two different release media: simulating gastric $(\mathrm{HCl})$ and intestinal conditions (PBS). The dissolution kinetics of CUR powder was studied in both 
simulated gastric and intestinal conditions (Figure 9C). A CUR burst release from the MPs can be observed during the first hour. During this time around $50 \%$ of the loaded CUR was released. After this initial burst, CUR is released in a sustained way during the next 6 hours. On the other hand, during the first hour around $60-70 \%$ of the pure CUR was dissolved in simulated intestinal and gastric conditions. Subsequently, all the free CUR was dissolved during the next 2 hours in both media.

It is important to note that MPs provided between 3 and 4 hours of sustained release when compared to the dissolution of the free drug. This behaviour is consistent with the obtained CUR release from the hydrogels (Figure 7). The initial burst release can be due to the presence of CUR inside the MPs that is not interacting with the hydrophobic T85 domains. Nevertheless, it can be seen that, despite the $\mathrm{pH}$-depending swelling of the hydrogels, the release does not show a biphasic pattern. This indicates that the release mechanism is governed by the interactions of the hydrophobic drug with the hydrogel network containing hydrophobic moieties. In addition, it can be seen that the profile is heavily influenced by the particle size as the MPs are able to release all the CUR cargo in 7 hours.

The results from this experiment showed that the hydrogel particle size strongly influences the release kinetics. Hydrogel films were able to provide up to 30 days of release while MPs were able to provide only 7 hours of release.

\section{Discussion}

Drug delivery systems based on hydrogels, have been limited to carrying hydrophilic drugs rather than hydrophobic drugs (Hoare and Kohane 2008; McKenzie, et al. 2015). The main reason for this is the limited quantity/homogeneity of loaded hydrophobic drugs in hydrogel matrices (McKenzie, et al. 2015). During the past decade, numerous research has been published describing strategies to improve hydrophobic drug loading/release capabilities of hydrogels (Gu, et al. 2016; McKenzie, et al. 2015). This is a key challenge as over $40 \%$ of the marketed drugs and about $60 \%$ of the therapeutic compounds at the research and development stage are hydrophobic compounds (Fahr and Liu 2007; Gu, et al. 2016). Consequently, there is a need for the development of new types of hydrogels for hydrophobic drug delivery.

Combined strategies have been used to improve the compatibility of hydrogel materials with hydrophobic drugs, including the insertion of cyclodextrins, hydrophobic moieties, nanoparticles or micelles within the hydrogel structure (Concheiro and Alvarez-Lorenzo 2013; Gu, et al. 2016; Jalalvandi, et al. 2017; Machín, et al. 2013; McKenzie, et al. 2015). These systems provided 
controlled drug release over different periods of time, ranging from a few hours to several days (Gu, et al. 2016). The type of application will be dependent on the type of hydrogel used and the drug release process.

In the present work, we described the synthesis of hydrogels containing T85 for hydrophobic drug delivery. Polysorbates such as T85 and GAN have been used extensively to prepare pharmaceutical formulations (Agüeros, et al. 2011; Kaur and Mehta 2017; Larrañeta, et al. 2015). These compounds have also been shown to be non-toxic (Ojer, et al. 2013; Sigward, et al. 2013). Therefore, they are good candidates to be used in this study.

Hydrogels were obtained through an esterification reaction carried out in solid state. The synthetic does not require the use of any type of organic solvent and the only by-product generated after the esterification reaction is water. Synthetic processes that do not require the use of organic solvents and reagents that can present toxicity problems are ideal for biological applications. Consequently, the described synthetic process can be considered safe and environmental-friendly. FTIR measurements (Figure 3) and the swelling of the resulting hydrogels (Figure 1) confirmed the crosslinking reaction. A similar procedure has been used before to prepare GAN hydrogels, however these materials were used mainly for the delivery of hydrophilic drugs as the selected crosslinkers were PEG or poly(vinyl alcohol) (Calo, et al. 2016b; Raj Singh, et al. 2010).

One of the main limitations of hydrogels for pharmaceutical applications is their inability to control the drug release over longer periods of time (Hoare and Kohane 2008). The high water content and large pore sizes of these materials often result in relatively rapid drug release. GAN/T85 hydrogels do not present this limitation due to the presence of hydrophobic moieties inside the hydrogel. CUR was selected as model hydrophobic drug to evaluate the drug loading/release capabilities of the hydrogels. It can be seen that the presence of hydrophobic moieties within the hydrogels improved significantly the loading of the molecule inside the materials. Furthermore, the presence of T85 in the hydrogels provided sustained release up to 30 days.

Hydrogel-based systems have been used before as platforms to delivery CUR (Alibolandi, et al. 2017; Gong, et al. 2013; Li, et al. 2012; Sun, et al. 2014). All these systems were designed as hydrogels containing CUR nanoparticles/nanosuspensions. For this purpose, hydrogels? should be prepared around the preformed nanosuspensions. These systems were able to sustain CUR release over a period ranging between a few hours to 15 days. As the system described in the present paper already contains the hydrophobic domains inside the hydrogels, there is no need to include any type of CUR containing nanocarrier. These hydrogels also provided sustained release process over longer periods of time. 
The hydrogels described in this work demonstrate potential to be used as drug eluting implants, as they allow sustained releases over long periods of time. Examples of hydrogels used for this aim can be found in the literature (Gu, et al. 2015; Pitarresi, et al. 2013). The hydrogels can be implanted subcutaneously, using a simple surgical procedure, providing a prolonged release of the loaded drug. GAN/T85 hydrogels showed promising properties to be used as drug eluting implants. However, the non-degradable nature of the polymers forming the hydrogel need to be considered. Accordingly, this type of material should be surgically implanted and removed after exhausting its drug cargo.

The proposed materials have potential for other drug delivery applications, such as oral drug delivery systems. Due to the presence of GAN in the structure, the prepared hydrogels showed good mucoadhesive properties and $\mathrm{pH}$-dependant swelling (Figure 8). Hydrogel MPs containing CUR were used as drug delivery systems. The results showed that CUR release was not influenced by the $\mathrm{pH}$ (Figure 9). In addition, the size reduction allowed to accelerate drug release. The MPs were able to release their cargos in 7 hours. The release profiles obtained for the MPs are more suitable for oral drug delivery applications than the extended release profiles obtained for the hydrogels (Figure 7). Interestingly, the release from the hydrogel MPs is not as sustained as the one obtained for the hydrogel films and it is close to the dissolution profile of the free drug. Consequently, the dimensions of the hydrogels should be chosen carefully as a function of the application. However, one of the advantages of the hydrogel MPs is their mucoadhesive properties that will expand the residence time of the system in the gastrointestinal tract.

CUR loaded MPs presented a burst release during the first hour. Further studies should evaluate alternative strategies to reduce this initial release. If the system is designed to be adhered to the intestinal mucosa and release its cargo there, the MPs should be encapsulated in a gastro-resistant capsule. An alternative solution will be to increase the particle size of the hydrogels or to use hydrogel films. Consequently, further studies should be performed to evaluate the influence of the particle size in the loading/release of hydrophobic compounds from this type of hydrogels.

\section{Conclusions}

The present paper describes the synthesis of hydrogels made of Gantrez and Tween 85 . The resulting hydrogel materials contained hydrophobic moieties, improving the compatibility of the materials with hydrophobic compounds. The synthetic process is simple and, as it does not require any toxic or organic solvents. Consequently, it can be considering safe and environmental-friendly. 
The obtained hydrogels were able to be successfully loaded with CUR, a hydrophobic model drug molecule, and to sustain the release of this compound over 30 days. Consequently, the material showed potential to be used for long term drug release applications, such as implants. Additionally, this type of hydrogels was tested for oral drug delivery applications, as the synthesized materials were mucoadhesive. Hydrogel MPs were prepared and tested for CUR release under simulated gastric and intestinal conditions. The results suggested that this type of material can be used for oral drug delivery but further work is required to optimize it.

\section{Acknowledgement}

The authors are thankful to Prof. R.F. Donnelly for his support during the experiments, Croda Europe Ltd for providing Tween ${ }^{\circledR} 85$ samples and to Ashland Specialties UK Ltd for providing Gantrez ${ }^{\circledR}$ S-97 samples. Additionally, Dr. Larraneta thanks Dr. M. Butler for her assistance during the manuscript writing.

\section{References}

Aggarwal, B.B., Sung, B., 2009. Pharmacological basis for the role of curcumin in chronic diseases: an age-old spice with modern targets. Trends Pharmacol. Sci., 30, 85-94. doi:

https://doi.org/10.1016/i.tips.2008.11.002.

Agüeros, M., Espuelas, S., Esparza, I., Calleja, P., Peñuelas, I., Ponchel, G., Irache, J.M., 2011. Cyclodextrin-poly(anhydride) nanoparticles as new vehicles for oral drug delivery. Expert Opin. Drug Deliv., 8, 721-734.

Agüeros, M., Ruiz-Gatón, L., Vauthier, C., Bouchemal, K., Espuelas, S., Ponchel, G., Irache, J.M., 2009. Combined hydroxypropyl- $\beta$-cyclodextrin and poly(anhydride) nanoparticles improve the oral permeability of paclitaxel. Eur. J. Pharm. Sci., 38, 405-413. doi:

https://doi.org/10.1016/j.ejps.2009.09.010.

Alibolandi, M., Mohammadi, M., Taghdisi, S.M., Abnous, K., Ramezani, M., 2017. Synthesis and preparation of biodegradable hybrid dextran hydrogel incorporated with biodegradable curcumin nanomicelles for full thickness wound healing. Int. J. Pharm., 532, 466-477. doi: https://doi.org/10.1016/j.ijpharm.2017.09.042.

Arbós, P., Wirth, M., Arangoa, M.A., Gabor, F., Irache, J.M., 2002. Gantrez AN as a new polymer for the preparation of ligand-nanoparticle conjugates. J. Control. Release, 83, 321-330.

Bajpai, S.K., Singh, S., 2006. Analysis of swelling behavior of poly(methacrylamide-co-methacrylic acid) hydrogels and effect of synthesis conditions on water uptake. React. Funct. Polym., 66, 431440. 
Boddupalli, B.M., Mohammed, Z.N.K., Nath, R.A., Banji, D., 2010. Mucoadhesive drug delivery system: An overview. J. Adv. Pharm. Technol. Res., 1, 381-387. doi: 10.4103/0110-5558.76436.

Branco, M.C., Schneider, J.P., 2008. Self-assembling materials for therapeutic delivery. Acta Biomater., 5, 817-831. doi: 10.1016/j.actbio.2008.09.018.

Calo, E., Barros, J., Ballamy, L., Khutoryanskiy, V.V., 2016a. Poly(vinyl alcohol)-Gantrez ${ }^{\circledR}$ AN cryogels for wound care applications. RSC Adv., 6, 105487-105494. doi: 10.1039/C6RA24573K.

Calo, E., Barros, J.M.S.d., Fernandez-Gutierrez, M., San Roman, J., Ballamy, L., Khutoryanskiy, V.V., 2016b. Antimicrobial hydrogels based on autoclaved poly(vinyl alcohol) and poly(methyl vinyl etheralt-maleic anhydride) mixtures for wound care applications. RSC Adv., 6, 55211-55219. doi:

10.1039/C6RA08234C.

Caló, E., Khutoryanskiy, V.V., 2015. Biomedical applications of hydrogels: A review of patents and commercial products. Eur. Polym. J., 65, 252-267. doi: http://dx.doi.org/10.1016/j.eurpolymj.2014.11.024.

Cappello, J., Crissman, J.W., Crissman, M., Ferrari, F.A., Textor, G., Wallis, O., Whitledge, J.R., Zhou, X., Burman, D., Aukerman, L., Stedronsky, E.R., 1998. In-situ self-assembling protein polymer gel systems for administration, delivery, and release of drugs. J. Control. Release, 53, 105-117. doi: https://doi.org/10.1016/S0168-3659(97)00243-5.

Çaykara, T., Kiper, S., Demirel, G., 2006. Network parameters and volume phase transition behavior of poly(N-isopropylacrylamide) hydrogels. J. Appl. Polym. Sci., 101, 1756-1762.

Concheiro, A., Alvarez-Lorenzo, C., 2013. Chemically cross-linked and grafted cyclodextrin hydrogels: From nanostructures to drug-eluting medical devices. Adv. Drug Deliv. Rev., 65, 1188-1203. doi: https://doi.org/10.1016/i.addr.2013.04.015.

Costa, P., Manuel, J., Lobo, S., Sousa Lobo, J.M., 2001. Modeling and comparison of dissolution profiles. European journal of pharmaceutical sciences, 13, 123-33. doi: 10.1016/S09280987(01)00095-1.

Crini, G., Morcellet, M., 2002. Synthesis and applications of adsorbents containing cyclodextrins. J. Sep. Sci., 25, 789-813.

Crini, G., 2003. Studies on adsorption of dyes on beta-cyclodextrin polymer. Bioresour. Technol., 90, 193-198.

Donnelly, R.F., Singh, T.R.R., Garland, M.J., Migalska, K., Majithiya, R., McCrudden, C.M., Kole, P.L., Mahmood, T.M.T., McCarthy, H.O., Woolfson, A.D., 2012. Hydrogel-Forming Microneedle Arrays for Enhanced Transdermal Drug Delivery. Adv. Funct. Mater., 22, 4879-4890.

Fahr, A., Liu, X., 2007. Drug delivery strategies for poorly water-soluble drugs. Expert Opin. Drug Deliv., 4, 403-416.

Flory, P.J., Rehner, J.J., 1943. Statistical Mechanics of Cross-Linked Polymer Networks I. Rubberlike Elasticity. J. Chem. Phys., 11, 512-520. doi: 10.1063/1.1723791. 
García-Zubiri, I.X., González-Gaitano, G., Isasi, J.R., 2009. Sorption models in cyclodextrin polymers: Langmuir, Freundlich, and a dual-mode approach. J. Colloid Interface Sci, 337, 11-18.

Gong, C.Y., Wu, Q.J., Wang, Y., Zhang, D.D., Luo, F., Zhao, X., Wei, Y.Q., Qian, Z.Y., 2013. A biodegradable hydrogel system containing curcumin encapsulated in micelles for cutaneous wound healing. Biomaterials, 34, 6377-6387. doi: https://doi.org/10.1016/j.biomaterials.2013.05.005.

Gu, B., Wang, Y., Burgess, D.J., 2015. In vitro and in vivo performance of dexamethasone loaded PLGA microspheres prepared using polymer blends. Int. J. Pharm., 496, 534-540. doi: https://doi.org/10.1016/j.ijpharm.2015.10.056.

Gu, D., O'Connor, A.J., Qiao, G.G.H., Ladewig, K., 2016. Hydrogels with smart systems for delivery of hydrophobic drugs. Expert Opin. Drug Deliv., 1-17. doi: 10.1080/17425247.2017.1245290.

Hoare, T., Kohane, D., 2008. Hydrogels in drug delivery: Progress and challenges. Polymer, 49, 19932007.

Hoerr, C.W., Harwood, H.J., 1952. The Solubilities of Oleic and Linoeic Acids in Common Organic Solvents. J. Phys. Chem., 56, 1068-1073. doi: 10.1021/j150501a008.

Jalalvandi, E., Hanton, L.R., Moratti, S.C., 2017. Schiff-base based hydrogels as degradable platforms for hydrophobic drug delivery. Eur. Polym. J., 90, 13-24. doi:

https://doi.org/10.1016/i.eurpolymi.2017.03.003.

Kaur, G., Mehta, S.K., 2017. Developments of Polysorbate (Tween) based microemulsions: Preclinical drug delivery, toxicity and antimicrobial applications. Int. J. Pharm., 529, 134-160. doi: https://doi.org/10.1016/j.ijpharm.2017.06.059.

Kharat, M., Du, Z.Z.,G., McClements, D.J., 2017. Physical and Chemical Stability of Curcumin in Aqueous Solutions and Emulsions: Impact of pH, Temperature, and Molecular Environment. J. Agric. Food Chem., 65, 1525-1532. doi: 10.1021/acs.jafc.6b04815.

Kopecek, J., 2009. Hydrogels: From soft contact lenses and implants to self-assembled nanomaterials. J. Polym. Sci. A., 47, 5929-5946. doi: 10.1002/pola.23607.

Larrañeta E, Martínez-Ohárriz C, Vélaz I, Zornoza A, Machín R, Isasi JR, 2014. In Vitro release from reverse poloxamine/ $\alpha$-cyclodextrin matrices: modelling and comparison of dissolution profiles. J. Pharm. Sci., 103, 197-206.

Larrañeta, E., Henry, M., Irwin, N.J., Trotter, J., Perminova, A.A., Donnelly, R.F., 2018. Synthesis and characterization of hyaluronic acid hydrogels crosslinked using a solvent-free process for potential biomedical applications. Carbohydr. Polym., 181, 1194-1205. doi: https://doi.org/10.1016/j.carbpol.2017.12.015.

Larrañeta, E., Isasi, J.R., 2014. Non-covalent hydrogels of cyclodextrins and poloxamines for the controlled release of proteins. Carbohydr. Polym., 102, 674-681.

Larrañeta, E., Lutton, R.E.M., Brady, A.J., Vicente-Pérez, E.M., Woolfson, A.D., Thakur, R.R.S., Donnelly, R.F., 2015. Microwave-Assisted Preparation of Hydrogel-Forming Microneedle Arrays for Transdermal Drug Delivery Applications. Macromol. Mater. Eng., 300, 586-595. doi:

10.1002/mame.201500016. 
Larrañeta, E., Isasi, J.R., 2012. Self-assembled Supramolecular Gels of Reverse Poloxamers and Cyclodextrins. Langmuir, 28, 12457-12462. doi: 10.1021/la3024452.

Li, X., Chen, S., Zhang, B., Li, M., Diao, K., Zhang, Z., Li, J., Xu, Y., Wang, X., Chen, H., 2012. In situ injectable nano-composite hydrogel composed of curcumin, $\mathrm{N}, \mathrm{O}$-carboxymethyl chitosan and oxidized alginate for wound healing application. Int. J. Pharm., 437, 110-119. doi: https://doi.org/10.1016/i.ijpharm.2012.08.001.

Machín, R., Isasi, J.R., Vélaz, I., 2013. Hydrogel matrices containing single and mixed natural cyclodextrins. Mechanisms of drug release. Eur. Polym. J., 49, 3912-3920.

Machín, R., Isasi, J.R., Vélaz, I., 2011. ß-Cyclodextrin hydrogels as potential drug delivery systems. Carbohydr. Polym.

McKenzie, M., Betts, D., Suh, A., Bui, K., Kim, L.D., Cho, H., 2015. Hydrogel-Based Drug Delivery Systems for Poorly Water-Soluble Drugs. Molecules, 20, 20397-20408.

Mohan, P.R.K., Sreelakshmi, G., Muraleedharan, C.V., Joseph, R., 2012. Water soluble complexes of curcumin with cyclodextrins: Characterization by FT-Raman spectroscopy. Vib. Spectrosc., 62, 77-84. doi: https://doi.org/10.1016/j.vibspec.2012.05.002.

Moreno, E., Schwartz, J., Larrañeta, E., Nguewa, P.A., Sanmartín, C., Agüeros, M., Irache, J.M., Espuelas, S., 2014. Thermosensitive hydrogels of poly(methyl vinyl ether-co-maleic anhydride) Pluronic $\left({ }^{\circledR}\right)$ F127 copolymers for controlled protein release. Int. J. Pharm., 459, 1-9.

Ojer, P., Neutsch, L., Gabor, F., Irache, J.M., López de Cerain, A., 2013. Cytotoxicity and cell interaction studies of bioadhesive poly(anhydride) nanoparticles for oral antigen/drug delivery. J. Biomed. Nanotechnol., 9, 1891-1903.

Parthiban, S., Meenakshisundaram, S., Bhagavannarayana, G., 2006. Enhancement of crystalline perfection by organic dopants in ZTS, ADP and KHP crystals as investigated by high-resolution XRD and SEM. J Appl. Crystallogr., 39, 784-790.

Peppas, N.A., Bures, P., Leobandung, W., Ichikawa, H., 2000. Hydrogels in pharmaceutical formulations. Eur. J. Pharm. Biopharm., 50, 27-46.

Peppas, N., Hilt, J., Khademhosseini, A., Langer, R., 2006. Hydrogels in biology and medicine: From molecular principles to bionanotechnology. Adv Mater, 18, 1345-1360.

Pitarresi, G., Palumbo, F.S., Calascibetta, F., Fiorica, C., Di Stefano, M., Giammona, G., 2013. Medicated hydrogels of hyaluronic acid derivatives for use in orthopedic field. Int. J. Pharm., 449, 8494. doi: https://doi.org/10.1016/j.ijpharm.2013.03.059.

Raj Singh, T.R., McCarron, P.A., Woolfson, A.D., Donnelly, R.F., 2009. Investigation of swelling and network parameters of poly(ethylene glycol)-crosslinked poly(methyl vinyl ether-co-maleic acid) hydrogels. Eur. Polym. J., 45, 1239-1249.

Raj Singh, T.R., Woolfson, A.D., Donnelly, R.F., Raj Singh, T.R., Woolfson, A.D., Donnelly, R.F., Raj Singh TR, Woolfson AD, Donnelly RF, 2010. Investigation of solute permeation across hydrogels composed of poly(methyl vinyl ether-co-maleic acid) and poly(ethylene glycol). J. Pharm. Pharmacol., $62,829-837$. 
Ritger, P.L., Peppas, N., 1987. A simple equation for description of solute release I. Fickian and NonFickian release from non-swellable devices in the form of slabs, spheres, cylinders or discs. J.

Controlled Release, 5, 23-36.

Sclavons, M., Franquinet, P., Carlier, V., Verfaillie, G., Fallais, I., Legras, R., Laurent, M., Thyrion, F.C., 2000. Quantification of the maleic anhydride grafted onto polypropylene by chemical and viscosimetric titrations, and FTIR spectroscopy. Polymer, 41, 1989-1999. doi:

http://dx.doi.org/10.1016/S0032-3861(99)00377-8.

Sigward, E., Mignet, N., Rat, P., Dutot, M., Muhamed, S., Guigner, J.M., Scherman, D., Brossard, D., Crauste-Manciet, S., 2013. Formulation and cytotoxicity evaluation of new self-emulsifying multiple W/O/W nanoemulsions. Int. J. Nanomedicine, 8, 611-625. doi: 10.2147/IJN.S35661.

Solak, E.N., Alawani, N., Wesdemiotis, C., 2014. Characterization of polysorbate 85, a nonionic surfactant, by liquid chromatography vs. ion mobility separation coupled with tandem mass spectrometry. Anal. Chim. Acta., 808, 83-93. doi: https://doi.org/10.1016/i.aca.2013.07.026.

Sun, Y., Du, L., Liu, Y., Li, X., Li, M., Jin, Y., Qian, X., 2014. Transdermal delivery of the in situ hydrogels of curcumin and its inclusion complexes of hydroxypropyl- $\beta$-cyclodextrin for melanoma treatment. Int. J. Pharm., 469, 31-39. doi: https://doi.org/10.1016/j.ijpharm.2014.04.039. 

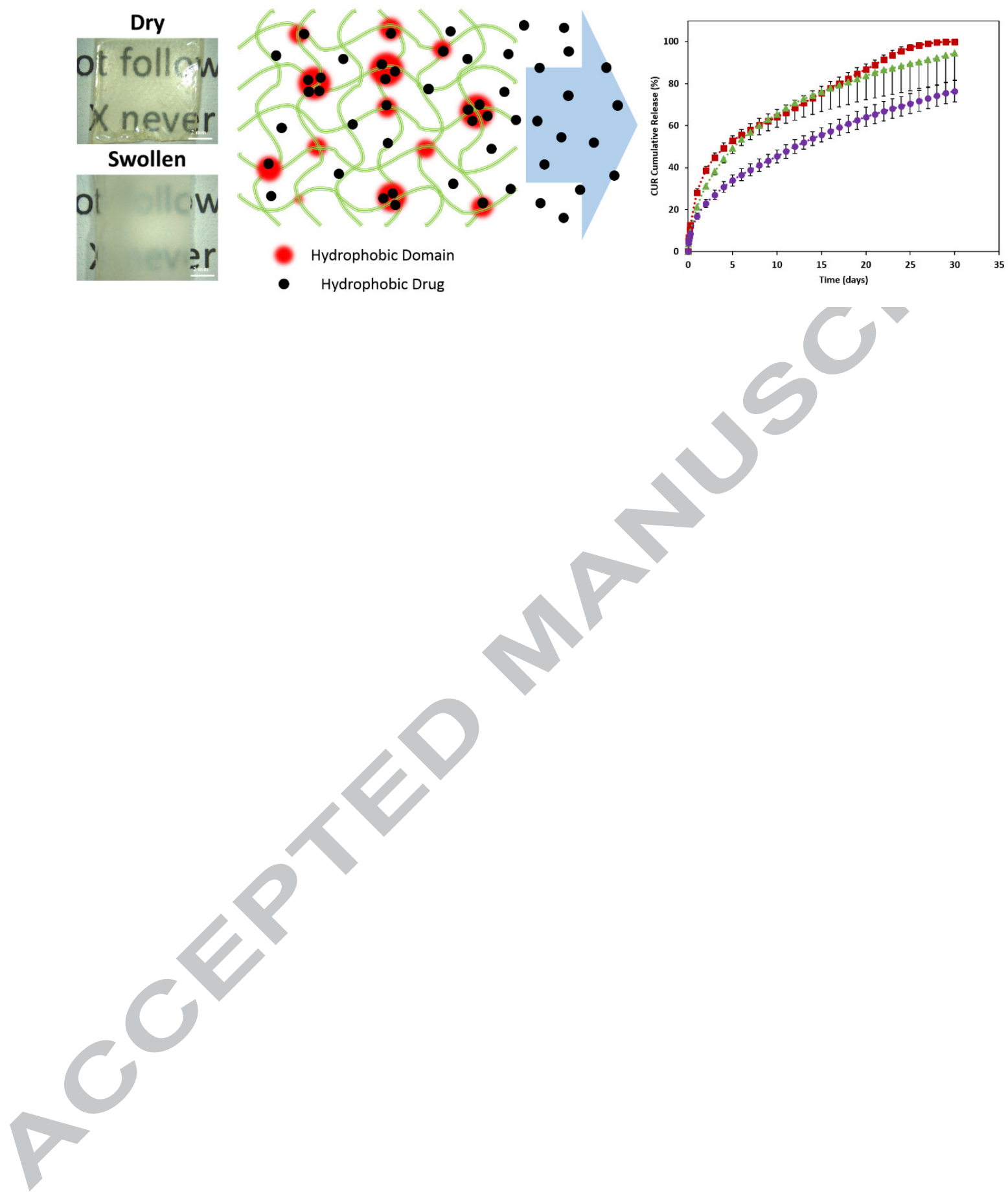
Figure 1. Size distribution obtained for different aqueous solutions containing different concentrations of Tween 85 (A). Swelling profiles of the synthesized hydrogels (B). Maximum swelling of the synthesized hydrogels in different organic solvents (C). Maximum swelling of the synthesized hydrogels as a function of the relative polarity of the solvent (D).

Figure 2. SEM images of swollen and freeze-dried hydrogels.

Figure 3. FTIR spectra of crosslinked and non-crosslinked (NC) P2 and T3 (A). FTIR spectra of the P2, $\mathrm{T} 2, \mathrm{~T} 3$ and $\mathrm{T} 5$ after the crosslinking process.

Figure 4. XRD patterns of $P 2, T 2, T 3$ and T5 (A). The full width at half maximum (FWHM) of the XRD peak of $\mathrm{P} 2, \mathrm{~T} 2, \mathrm{~T} 3$ and $\mathrm{T} 5$ hydrogels (B).

Figure 5. Images of T85/GAN hydrogels in their dry and swollen state.

Figure 6. Chemical structure of CUR (A). FTIR spectra of CUR and the hydrogels before and after being loaded with CUR (B). DSC of CUR and CUR loaded hydrogels. Exo Up.

Figure 7. CUR cumulative release from different hydrogels. Graphs shows the amount ( $A$ and $B$ ) and the percentage (C) of CUR released as a function of time. CUR released from P2 hydrogels (B). Percentage of CUR powder dissolved as a function of time (D).

Figure 8. Mucoadhesion force for the hydrogels (A). Swelling profiles of $\mathrm{T} 2$ and $\mathrm{T} 3$ as at $\mathrm{pH} 7.3$ and $\mathrm{pH} 1.2$.

Figure 9. SEM images of T2 MPs (A). CUR release profile from T2 MPs in simulated gastric $(\mathrm{HCl})$ and intestinal media (PBS) (B). Percentage of CUR powder dissolved in PBS as a function of time (C). 

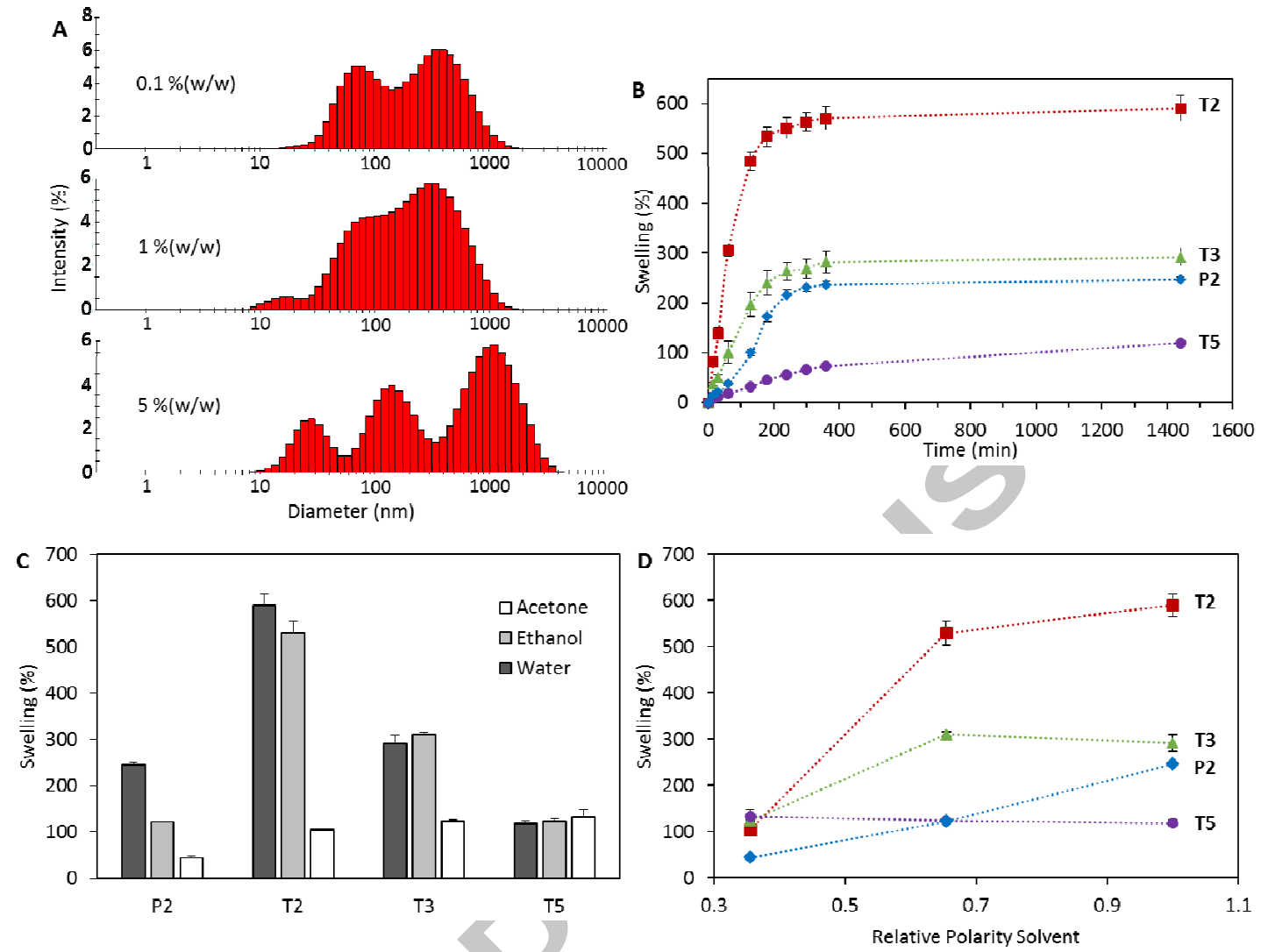

Figure 1 
P2
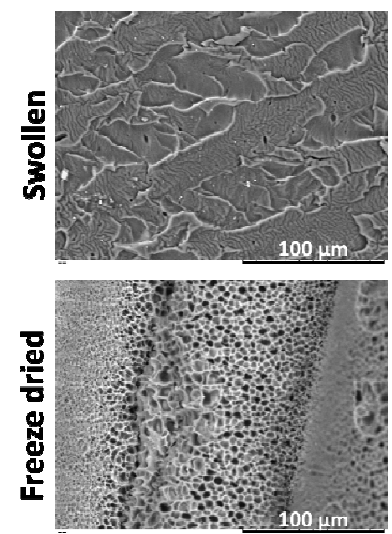

T3
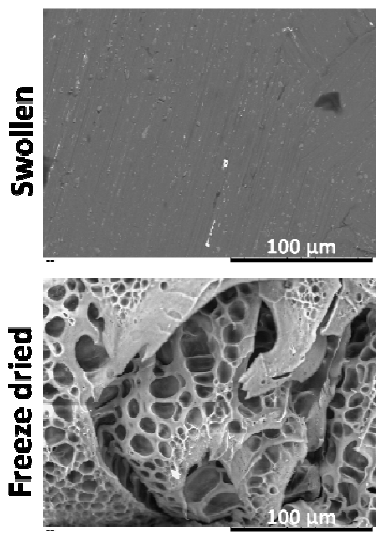

Figure 2
T2
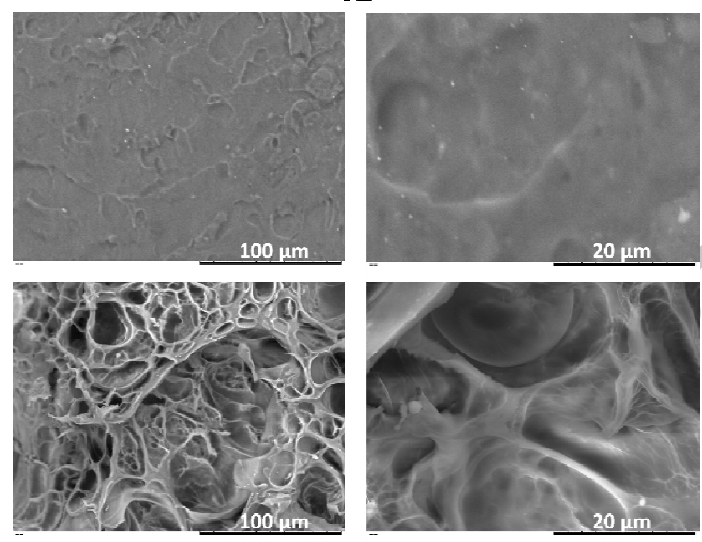

T5
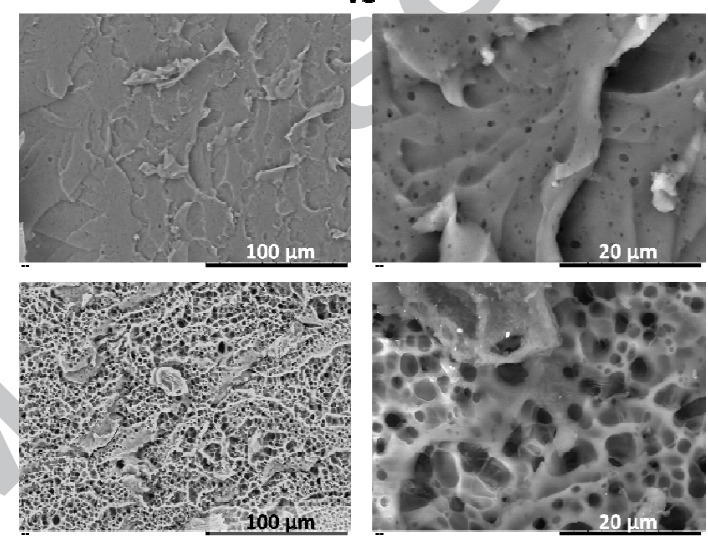

wo $1004 \mathrm{~m}$
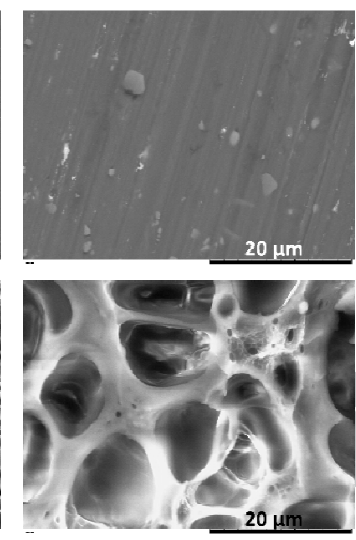

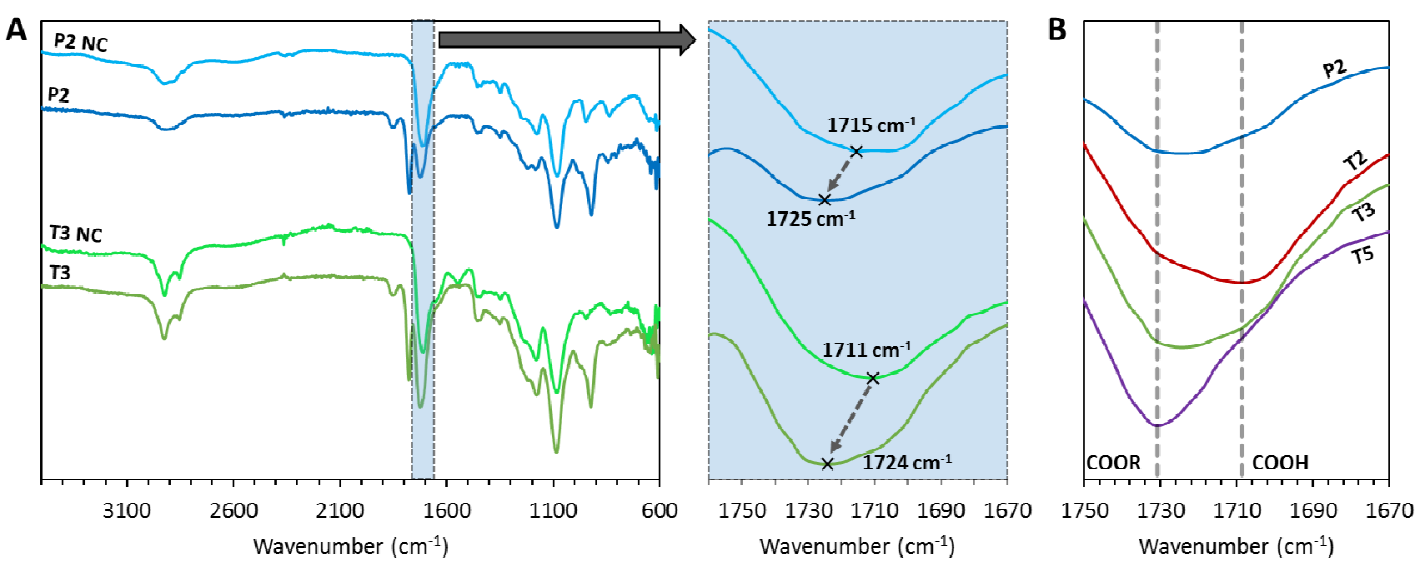

Figure 3 
A
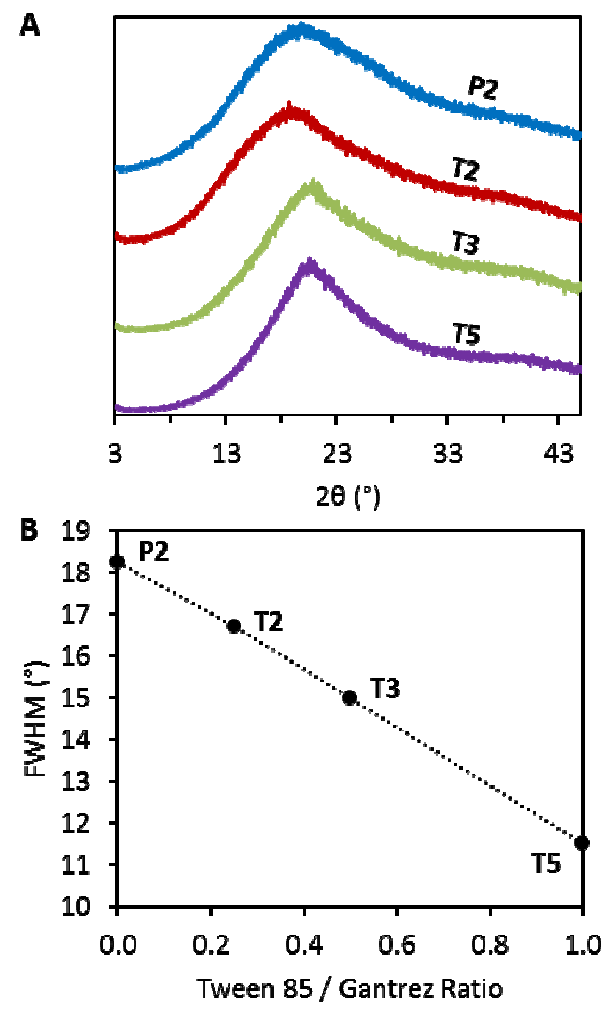

Figure 4 


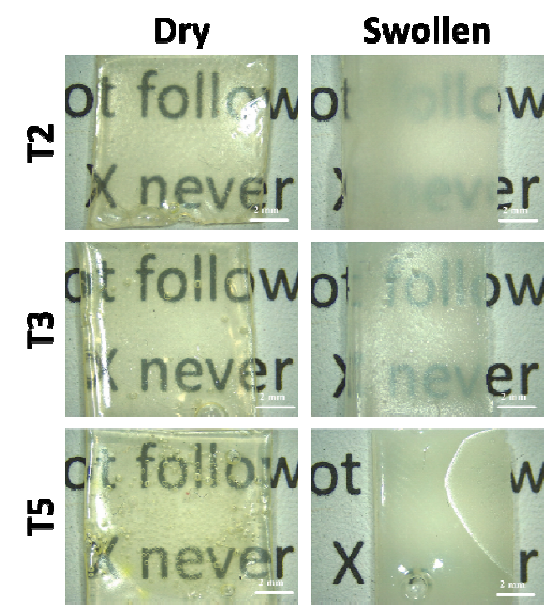

Figure 5 

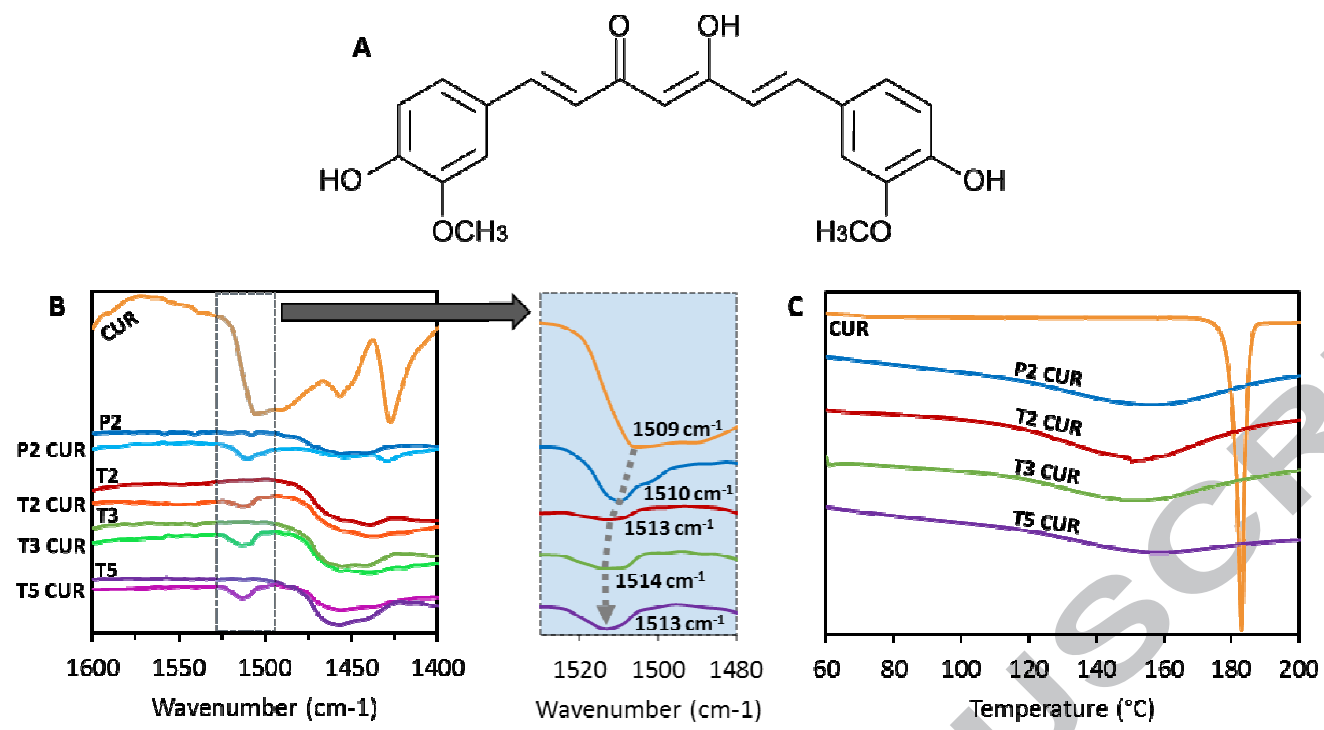

Figure 6 

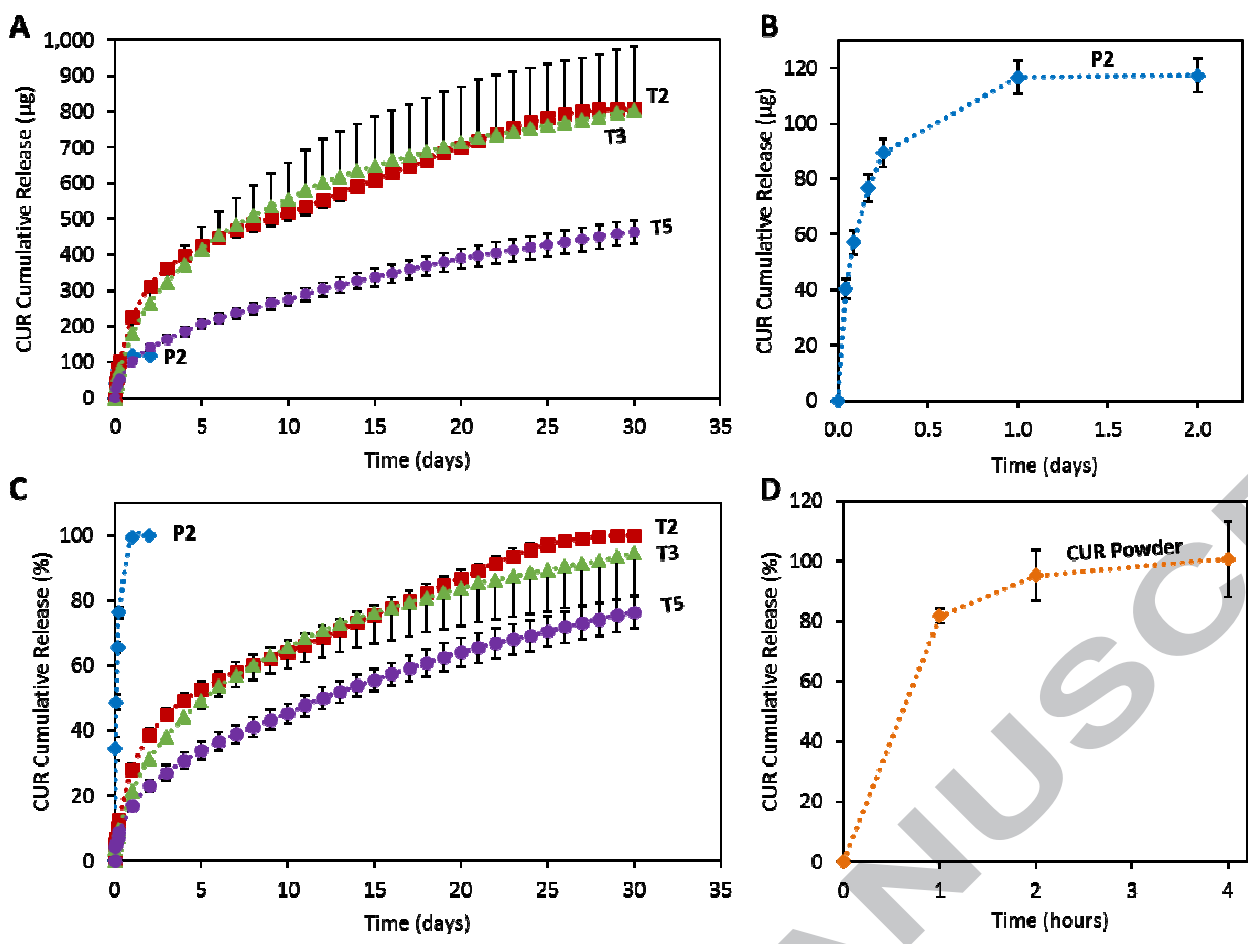

Figure 7 

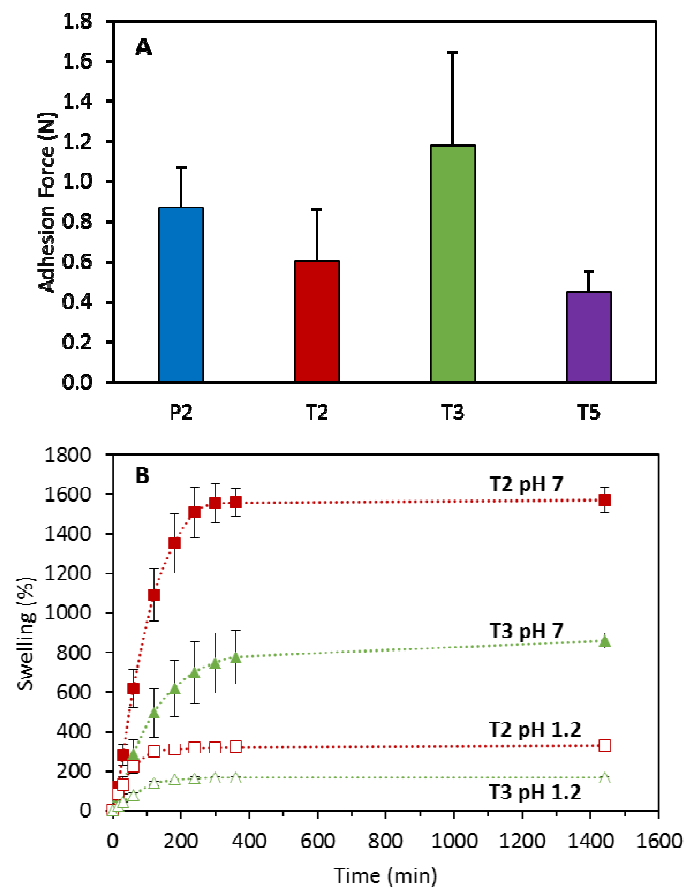

Figure 8 

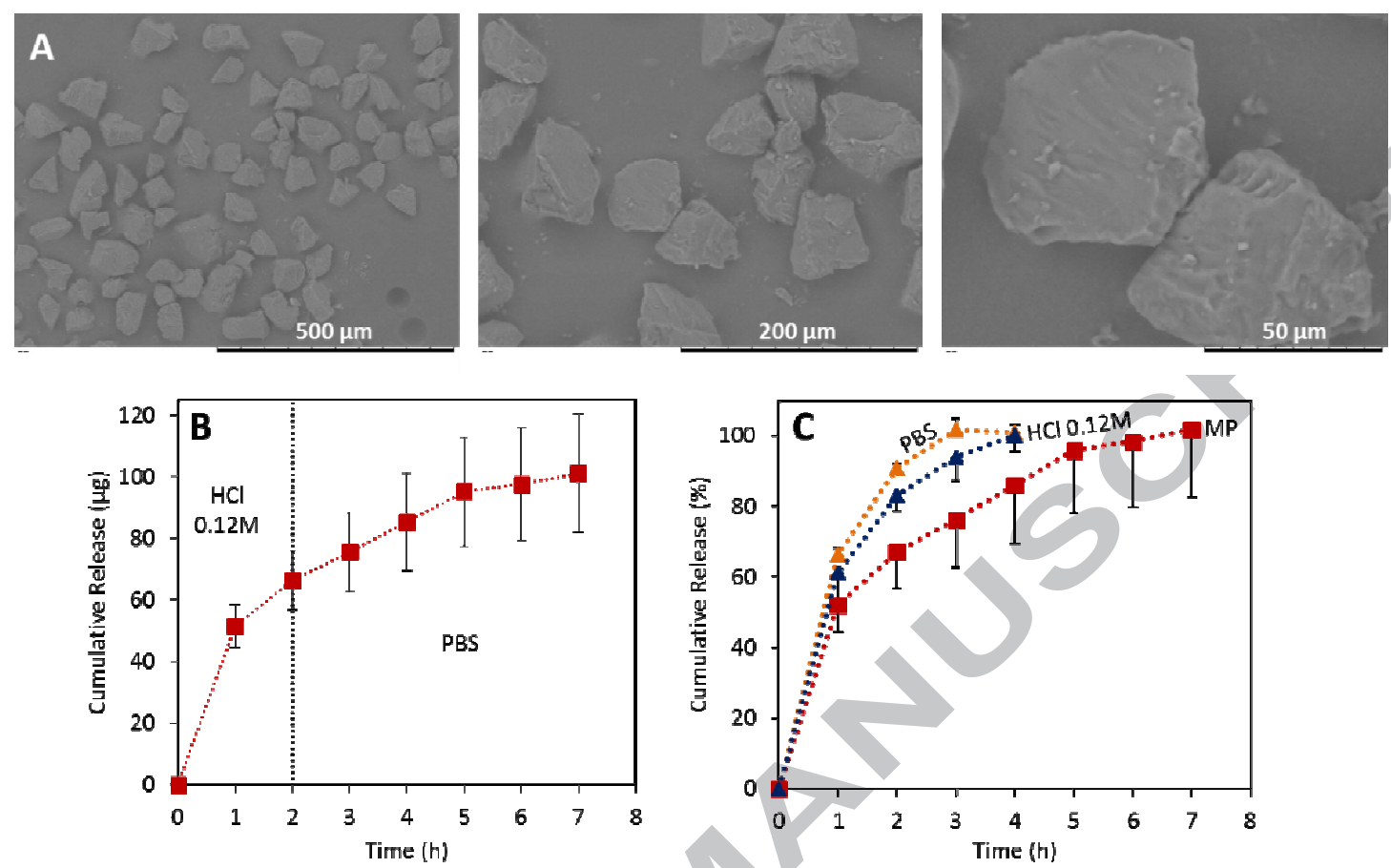

Figure 9 
Table 1. Composition of the aqueous blends used to prepare GAN/T85

\begin{tabular}{ccccc}
\hline \multirow{2}{*}{ Hydrogel } & \multicolumn{3}{c}{ Initial Mixture Composition } & \multirow{2}{*}{ T85/GAN } \\
\cline { 2 - 4 } & GAN (\%) (w/w) & T85 (\%) (w/w) & PEG (\%) (w/w) & \\
\hline P2 & 20.0 & - & 7.5 & $27 / 73$ \\
T2 & 20.0 & 5.0 & - & $20 / 80$ \\
T3 & 20.0 & 10.0 & - & $33 / 67$ \\
T5 & 20.0 & 20.0 & - & $50 / 50$ \\
\hline
\end{tabular}


Table 2. Network parameters obtained for the hydrogels

\begin{tabular}{ccccc}
\hline Hydrogel & $\phi$ & $\mathrm{M}_{\mathrm{c}}$ (Equi) $(\mathrm{kDa})$ & $\chi$ & $\mathrm{V}_{\mathrm{e}} \times 10^{-19}$ \\
\hline P2 & 0.26 & 9.40 & 0.59 & 7.44 \\
T2 & 0.14 & 101.58 & 0.55 & 0.64 \\
T3 & 0.25 & 10.16 & 0.58 & 6.20 \\
T5 & 0.48 & 0.58 & 0.66 & 94.84 \\
\hline
\end{tabular}


Table 3. CUR loading obtained for all the hydrogels.

\begin{tabular}{cc}
\hline Hydrogel & CUR loading $(\mathrm{mg} / \mathrm{g})$ \\
\hline P2 & $3.5 \pm 0.1$ \\
T2 & $26.6 \pm 0.5$ \\
T3 & $36.0 \pm 0.3$ \\
T5 & $35.7 \pm 1.4$ \\
\hline
\end{tabular}


Table 4. Values of dissolution efficiency (DE), mean dissolution Time (MDT), difference $\left(f_{1}\right)$ and similarity factors $\left(f_{2}\right)$ obtained for the release curves of the hydrogels.

\begin{tabular}{ccccc}
\hline Hydrogel & DE (\%) & MDT (days) & $f_{1}$ & $f_{2}$ \\
\hline P2 & 89.35 & 0.21 & $>15$ vs. all hydrogels & $<50$ vs. all hydrogels \\
T2 & 73.23 & 8.03 & 5.4 vs. T3 & 56.1 vs. T3 \\
& & & $>15$ vs the other hydrogels & $<50$ vs the other hydrogels \\
T3 & 70.17 & 7.75 & 5.4 vs. T2 & 56.1 vs. T2 \\
& & & $>15$ vs. the other hydrogels & $<50$ vs the other hydrogels \\
T5 & 52.53 & 9.40 & $>15$ vs. all hydrogels & $<50$ vs. all hydrogels \\
\hline
\end{tabular}


Table 5. Results of fitting the release of CUR from different hydrogels to Korsmeyer-Peppas and Higuchi models.

\begin{tabular}{|c|c|c|c|c|c|c|c|c|}
\hline \multirow[b]{2}{*}{ Hydrogel } & \multirow{2}{*}{$\begin{array}{c}\text { Range }^{1} \\
\text { (days) }\end{array}$} & \multicolumn{3}{|c|}{ Korsmeyer-Peppas Model } & \multicolumn{2}{|c|}{ Higuchi Model } & \multirow{2}{*}{$\begin{array}{r}- \text { Range }^{2} \\
\text { (days) }\end{array}$} & Zero Order Model \\
\hline & & $\begin{array}{c}\mathrm{k}_{\mathrm{KP}} \\
\left(\text { days }^{-n}\right)\end{array}$ & $\mathrm{n}$ & $\mathrm{R}^{2}$ & $\begin{array}{c}k_{\mathrm{H}} \\
\left(\text { days }^{-1 / 2}\right)\end{array}$ & $R^{2}$ & & $\begin{array}{l}\mathrm{k}_{\mathrm{zo}} \cdot 10^{2} \\
\left(\text { days }^{-1}\right)\end{array}$ \\
\hline P2 & $0-2$ & $1.49 \pm 0.05$ & $0.45 \pm 0.02$ & 0.999 & $1.64 \pm 0.02$ & 0.998 & - & - \\
\hline $\mathrm{T} 2$ & $0-7$ & $0.26 \pm 0.01$ & $0.43 \pm 0.02$ & 0.991 & $0.24 \pm 0.01$ & 0.983 & - & - \\
\hline $\mathrm{T} 2^{3}$ & $0-3$ & $0.27 \pm 0.01$ & $0.50 \pm 0.02$ & 0.997 & $0.27 \pm 0.01$ & 0.997 & $5-24$ & 0.010 .999 \\
\hline T3 & $0-7$ & $0.21 \pm 0.01$ & $0.52 \pm 0.01$ & 0.999 & $0.22 \pm 0.01$ & 0.999 & - & \\
\hline T5 & $0-17$ & $0.16 \pm 0.01$ & $0.45 \pm 0.01$ & 0.999 & $0.15 \pm 0.01$ & 0.996 & - & - \\
\hline
\end{tabular}

${ }^{1}$ Range: the release data within this range was used to apply the Korsmeyer-Peppas and Higuchi models ( $\leq 60 \%$ release).

${ }^{2}$ Range: the release data within this range was used to apply the Zero Order Model

${ }^{3}$ In this case $\mathrm{T} 2$ the release from $\mathrm{T} 2$ was separated in two sections to apply different release models: Korsmeyer-Peppas and Higuchi models for the first stage of the release (0-3 days) and Zero Order model for the last stage (5-24 days) 